\title{
Observational study of reactive ions and radicals in PDRs
}

\author{
A. Fuente ${ }^{1}$, A. Rodríguez-Franco ${ }^{2}$, S. García-Burillo ${ }^{1}$, J. Martín-Pintado ${ }^{3}$, and J. H. Black ${ }^{4}$ \\ 1 Observatorio Astronómico Nacional (IGN), Campus Universitario, Apdo. 1143, 28800 Alcalá de Henares (Madrid), Spain \\ 2 Universidad Complutense de Madrid, Av. Arcos de Jalón s/n, 28037 Madrid, Spain \\ 3 Departamento Física Molecular, Instituto de Estructura de la Materia, CSIC, Serrano 121, 28006 Madrid, Spain \\ ${ }^{4}$ Onsala Space Observatory, Chalmers University of Technology, 43992 Onsala, Sweden
}

Received 13 January 2003 / Accepted 18 April 2003

\begin{abstract}
We have carried out a survey of reactive ions $\left(\mathrm{CO}^{+}, \mathrm{HOC}^{+}, \mathrm{HC}^{18} \mathrm{O}^{+}, \mathrm{SO}^{+}\right)$and cyclopropenylidene $\left(\mathrm{C}_{3} \mathrm{H}_{2}\right)$ in three prototypical photodissociation regions (PDRs), the reflection nebula NGC 7023, the Orion Bar and the planetary nebula (PN) NGC 7027. The reactive ion $\mathrm{CO}^{+}$has been detected in all the targets with fractional abundances ranging from $\sim 10^{-11}$ to $\sim$ a few $10^{-9}$. Its spatial distribution in NGC 7023 and the Orion Bar show that $\mathrm{CO}^{+}$arises in the innermost part $\left(A_{v}<2\right.$ mag) of the PDR. In NGC 7027, the $\mathrm{CO}^{+}$lines shows an expansion velocity higher than that of the $\mathrm{CO}$ lines. This high expansion velocity is consistent with the $\mathrm{CO}^{+}$emission arising in the high velocity layer of neutral gas which is being accelerated by the ionized gas. Photochemistry determines the chemical composition of this layer.

The reactive ions $\mathrm{HOC}^{+}$and $\mathrm{SO}^{+}$have been detected in NGC 7023 and the Orion Bar. In both sources, the fractional abundance of $\mathrm{HOC}^{+}$is enhanced by a factor of $\sim 10$ towards the PDRs, with typical abundances, $X_{\mathrm{HOC}^{+}}=0.7-3 \times 10^{-11}$. This enhancement produces a decrease of the $\left[\mathrm{HCO}^{+}\right] /\left[\mathrm{HOC}^{+}\right]$abundance ratio towards the PDR. In fact, we have derived $\left[\mathbf{H C O}^{+}\right] /\left[\mathbf{H O C}^{+}\right] \sim$ 50-120 in NGC 7023, which is the lowest ratio measured thus far. $\mathrm{HOC}^{+}$and $\mathrm{SO}^{+}$have not been detected in NGC 7027. Interestingly, this is the source with the highest $\mathrm{CO}^{+}$abundance, $X_{\mathrm{CO}^{+}}=5 \times 10^{-9}$. This lack of detection is interpreted as due to the peculiar chemistry of C-rich PNs, in which the abundance of oxygenated molecules, in particular $\mathrm{H}_{2} \mathrm{O}$, is low.

We have detected cyclopropenylidene $\left(\mathrm{C}_{3} \mathrm{H}_{2}\right)$ in the three target PDRs. Similarly to the reactive ions, the abundance of $\mathrm{C}_{3} \mathrm{H}_{2}$ in NGC 7023 and the Orion Bar is a factor of 10-100 higher in the PDRs than in the foreground molecular cloud with peak values ranging from $10^{-10}$ to $10^{-9}$. In NGC 7027, we measured the maximum $\mathrm{C}_{3} \mathrm{H}_{2}$ abundance with a value of $\sim 10^{-8}$. Similarly to the case of $\mathrm{CO}^{+}$, the high expansion velocities of the $\mathrm{C}_{3} \mathrm{H}_{2}$ lines in NGC 7027 suggests that its emission arises in the neutral gas which is being accelerated by the ionized gas. Photodestruction of Polycyclic Aromatic Hydrocarbons (PAHs) is proposed to explain the enhanced $\mathrm{C}_{3} \mathrm{H}_{2}$ abundance in these PDRs.
\end{abstract}

Key words. ISM: clouds - ISM: abundances - ISM: molecules - ISM: individual objects: NGC 7023, the Orion Bar - planetary nebulae: individual: NGC 7027

\section{Introduction}

For the last five years we have carried out a study of the chemistry of photon-dominated regions (PDRs). Reactive ions are thought to be one of the best tracers of PDRs. These compounds react on virtually every collision with $\mathrm{H}$ and $\mathrm{H}_{2}$ and their abundances are expected to be negligible in the shielded parts of molecular clouds. Furthermore, they are destroyed rapidly by collisions with electrons. Only in the hot layers of PDRs, where the FUV field is only partially attenuated and maintains high densities of the parent species $\mathrm{C}^{+}$and $\mathrm{S}^{+}$, does the abundance of these ions become significant (Sternberg \& Dalgarno 1995).

Send offprint requests to: A. Fuente,

e-mail: a.fuente@oan.es
The molecular ion $\mathrm{CO}^{+}$was the first reactive ion discovered in the interstellar medium (Latter et al. 1993). So far, $\mathrm{CO}^{+}$has been detected in M17 SW, NGC 7027, the Orion Bar and NGC 7023 (Latter et al. 1993; Jansen et al. 1995; Störzer et al. 1995; Fuente \& Martín-Pintado 1997). It has been argued (Black 1998) that $\mathrm{CO}^{+}$arises in the hot $\mathrm{HI} / \mathrm{H}_{2}$ transition layer of PDRs and constitutes a potential diagnostic probe of this very interesting boundary layer.

In a possibly related development, the confirmation of interstellar $\mathrm{HOC}^{+}$by Ziurys \& Apponi (1995) indicates much lower values of the abundance ratio $\mathrm{HCO}^{+} / \mathrm{HOC}^{+}$in $\mathrm{PDRs}$ than expected. The abundance of this metastable isomer of $\mathrm{HCO}^{+}$ could be enhanced in PDRs resulting from high abundances of $\mathrm{C}^{+}$and $\mathrm{CO}^{+}$. Savage et al. (2000) proposed a correlation between the abundances of $\mathrm{HOC}^{+}$and $\mathrm{CO}^{+}$. 
Another chemically related ion that is expected to be abundant in the $\mathrm{HI} / \mathrm{H}_{2}$ transition layer is $\mathrm{SO}^{+}$. $\mathrm{SO}^{+}$was firstly detected in the interstellar medium towards the supernova remnant IC443 (Turner 1992) and proposed as a tracer of dissociative shocks. Later surveys of $\mathrm{SO}^{+}$that included dark clouds, star forming regions and high velocity molecular outflows suggest that $\mathrm{SO}^{+}$is not associated with shock chemistry (Turner 1994; Fuente et al. unpublished data). Chemical models predict a high $\mathrm{SO}^{+}$abundance in the $\mathrm{HI} / \mathrm{H}_{2}$ transition layer of PDRs (Sternberg \& Dalgarno 1995). So far, $\mathrm{SO}^{+}$has not been studied in these regions of high ionization rates.

In addition to the reactive ions, high abundances of hydrocarbon radicals like $\mathrm{C}_{2} \mathrm{H}$ and $\mathrm{c}-\mathrm{C}_{3} \mathrm{H}_{2}$ are observed towards PDRs (Fuente et al. 1993; Fossé et al. 2000). Fossé et al. (2000) compared the Aromatic Infrared Bands (AIBs) emission with the hydrocarbon abundances towards IC 63 and NGC 7023 and found that the high abundances of hydrocarbons are coincident with the emission of the Aromatic Infrared Bands (AIBs). They proposed that the destruction of Polycyclic Aromatic Hydrocarbons (PAHs) by UV radiation could produce high hydrocarbon abundances in PDRs.

In this paper we present observations of the molecular ions $\mathrm{CO}^{+}, \mathrm{HOC}^{+}, \mathrm{HC}^{18} \mathrm{O}^{+}$and $\mathrm{SO}^{+}$and cyclopropenylidene $\left(\mathrm{C}_{3} \mathrm{H}_{2}\right)$ in the prototypical photon-dominated regions (PDRs) NGC 7023, the Orion Bar and NGC 7027. These sources are selected to cover a wide range of physical conditions in PDRs (incident UV field, $G_{0}$, and density, $n$ ). Possible variations in the fractional abundances of these compounds from source to source can provide important information about the molecular chemistry in PDRs.

\section{Brief descriptions of the PDRs}

\subsection{NGC 7023}

NGC 7023 is a reflection nebula illuminated by the Herbig B3Ve star HD $200775\left(\mathrm{RA}(2000)=21^{\mathrm{h}} 01^{\mathrm{m}} 36^{\mathrm{s}} .9 \operatorname{Dec}(2000)=\right.$ $68^{\circ} 09^{\prime} 48^{\prime \prime}$ '3). HD 200775 is located in a cavity of the molecular cloud whose sharp edges perfectly delineate the optical nebula (Fuente et al. 1992, 1998; Rogers et al. 1995). Observations of CII and OI by Chokshi et al. (1988) reveal that a dense PDR is formed in the walls of this cavity with its peak located $50^{\prime \prime} \mathrm{NW}$ from the star (following the nomenclature of Fuente et al. 1997 we will refer to this position as the PDR-peak). Chokshi et al. (1988) carried out the first model for this region and estimated an intensity of the UV field of $G_{0}=2.4 \times 10^{3}$ in units of the Habing field and a density of $n=10^{4} \mathrm{~cm}^{-3}$. An interferometric image of the $\mathrm{HCO}^{+}$emission towards the PDR-peak show that this PDR has a filamentary structure in molecular emission with high density filaments $\left(n>10^{5} \mathrm{~cm}^{-3}\right)$ embedded in a more diffuse medium $\left(n \sim 10^{4} \mathrm{~cm}^{-3}\right)$ (Fuente et al. 1996). In Fig. 1, we show a scheme of the PDR associated with this nebula in which the spatial distribution of the atomic gas, the emission of the molecular gas in the $\mathrm{HCO}^{+} 1 \rightarrow 0$ line and the high density $\mathrm{HCO}^{+}$filaments are shown. Because of its edgeon geometry and proximity $(d \sim 440 \mathrm{pc})$, this PDR turned out to be one of the best sites to study the physical and chemical processes taking place in a PDR.

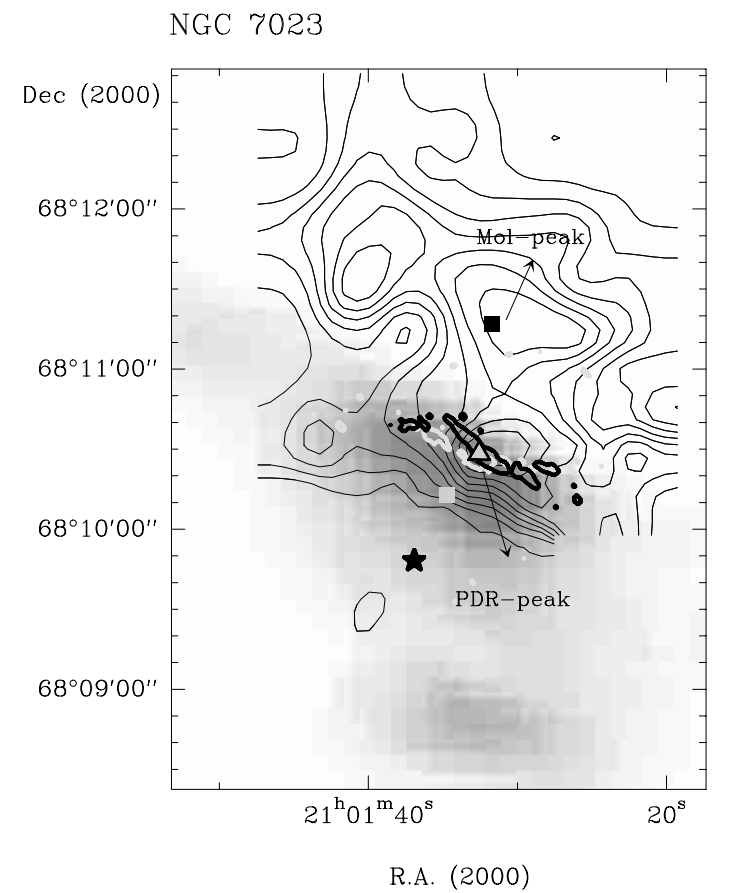

Fig. 1. Scheme of the reflection nebula NGC 7023. Contours of the integrated intensity of the $\mathrm{HCO}^{+} J=1 \rightarrow 0$ line are superposed on the HI column density image (gray scale) (Fuente et al. 1993; Fuente et al. 1996). The $\mathrm{HCO}^{+}$molecular filaments as observed with the Plateau de Bure interferometer are shown by black and white contours. These high density filaments are located at the interface between the atomic and molecular gas and are spatially coincident with the PDR-peak (white triangle). The observed positions are indicated in the figure by a star (the star position), two filled squares and a triangle (PDR-peak).

Fuente \& Martín-Pintado (1997) detected $\mathrm{CO}^{+}$towards the PDR-peak. It was the first, and so far only, detection of $\mathrm{CO}^{+}$in the vicinity of a Be star. However they did not detect $\mathrm{CO}^{+}$in a well-shielded clump of the adjacent molecular cloud (molecular-peak in the nomenclature of Fuente \& Martín-Pintado 1997). This lack of $\mathrm{CO}^{+}$emission cannot be due to different excitation conditions but to a different $\mathrm{CO}^{+}$abundance. They found that the $\mathrm{CO}^{+} / \mathrm{HCO}^{+}$ratio is at least 100 times larger in the PDR-peak than in the molecularpeak.

\subsection{The Orion Bar}

The HII region M 42 is located on the near side of the giant molecular cloud OMC1. Its proximity, $480 \mathrm{pc}$, makes this source a good target to study the interaction of HII regions with the molecular environment. M 42 is ionized by a group of OB stars known as the Trapezium stars. Optical images of the nebula show a bright bar located to the southwest of the Trapezium cluster. Observations of this so-called "Optical Bar" in radiocontinuum, vibrationally excited $\mathrm{H}_{2}$ (Hayashi et al. 1985), and at $3.3 \mu \mathrm{m}$ (Bregman et al. 1994; Giard et al. 1994) show that this bar is formed by the gas associated with an edge-on ionization front. The gas associated with this ionization front has been widely studied at all wavelengths (Parmar et al. 1991; Rodríguez-Franco et al. 1992; Omodaka et al. 1994; Tauber et al. 1994, 1995; Hogerheijde et al. 1995; White \& Sandell 1995; Fuente et al. 1996b) and modeled by 


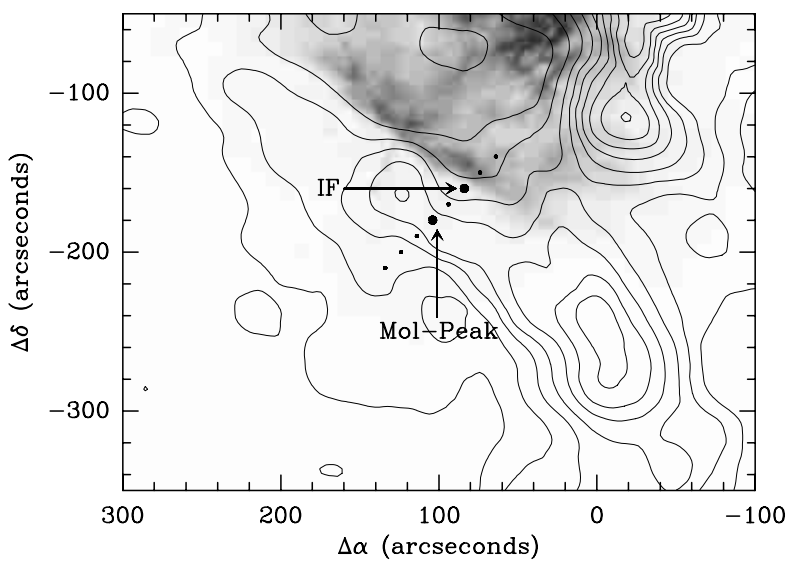

Fig. 2. Map of the integrated emission of the $\mathrm{CN} N=1 \rightarrow 0$ line towards the Orion Bar (Rodríguez-Franco et al. 1998) superposed to the image of the continuum at $20 \mathrm{~cm}$ (Yusef-Zadeh 1990). Dots represent the positions where data have been taken. The positions of the Molecular peak and the Ionitation front (IF) are indicated.

Burton et al. (1990) with a density $n=10^{5} \mathrm{~cm}^{-3}$ and an incident UV field $G_{0} \sim 4 \times 10^{4}$ in units of Habing field. Jansen et al. (1995) and Fuente et al. (1996b) found the trend that radicals such as $\mathrm{CN}$ and $\mathrm{C}_{2} \mathrm{H}$ and the reactive ion $\mathrm{CO}^{+}$have larger column densities close to the ionization front while other molecules are more abundant in the more shielded molecular cloud. In Fig. 2, we show a scheme of the region in which the map of the CN $N=1 \rightarrow 0$ line emission reported by RodríguezFranco et al. (1998) has been superposed on the radio continuum emission of the HII region (Yusef-Zadeh 1990). Apponi et al. (1999) detected $\mathrm{HOC}^{+}$towards the Orion Bar and determined a $\mathrm{HCO}^{+} / \mathrm{HOC}^{+}$ratio of $\sim 270$, the lowest found thus far in the Galaxy.

\subsection{NGC 7027}

NGC 7027 is a very young C-rich PN that left the AGB only $10^{3}$ years ago (Volk \& Kwok 1997). The temperature of the central star is $\sim 2 \times 10^{5} \mathrm{~K}$ (Latter et al. 2000). It has an extremely rich emission-line spectrum and is one of the most extensively studied objects in the sky. The gas in the inner $10^{\prime \prime}$ radius of the envelope is fully ionized and emits strong continuum emission. The ionized gas is surrounded by a molecular envelope with a size greater than 70" (Bieging et al. 1991). The PDR between the ionized and the molecular gas is traced by the emission of the vibrationally excited $\mathrm{H}_{2}$ (Cox et al. 1997) and present a bi-conical morphology with a size of $\sim 13^{\prime \prime}$. Reactive ions and radicals like $\mathrm{HCO}^{+}, \mathrm{CO}^{+}, \mathrm{CH}^{+}, \mathrm{CN}$, and $\mathrm{C}_{2} \mathrm{H}$ (Latter et al. 1993; Cernicharo et al. 1997; Hasegawa \& Kwok 2001) have been detected in this nebula. The observed lines of these species show line-widths wider than those of the CO lines (Hasegawa $\&$ Kwok 2001). Furthermore, the estimated size for the emitting region was $\sim 13^{\prime \prime}$, much smaller than that of the CO envelope. Hasegawa \& Kwok (2001) proposed that the emission of these molecules arise mainly in a layer of neutral gas that is being accelerated by the ionized gas.
Table 1. Observing frequencies and telescope parameters.

\begin{tabular}{lccc}
\hline \hline Line & Freq. (GHz) & beam & $\eta_{M B}$ \\
\hline $\mathrm{HOC}^{+} 1 \rightarrow 0$ & 89.4874 & $29^{\prime \prime}$ & 0.80 \\
$\mathrm{HOC}^{+} 3 \rightarrow 2$ & 268.451 & $9.5^{\prime \prime}$ & 0.50 \\
$\mathrm{HC}^{18} \mathrm{O}^{+} 1 \rightarrow 0$ & 85.1622 & $29^{\prime \prime}$ & 0.80 \\
$\mathrm{CO}^{+} 2,5 / 2 \rightarrow 1,3 / 2$ & 236.0625 & $10.5^{\prime \prime}$ & 0.50 \\
$\mathrm{CO}^{+} 2,3 / 2 \rightarrow 1,1 / 2$ & 235.7896 & $10.5^{\prime \prime}$ & 0.50 \\
$\mathrm{SO}^{+} 9 / 2 \rightarrow 7 / 2(\mathrm{e})$ & 208.5900 & $12^{\prime \prime}$ & 0.54 \\
$\mathrm{SO}^{+} 9 / 2 \rightarrow 7 / 2(\mathrm{f})$ & 208.9654 & $12^{\prime \prime}$ & 0.54 \\
$\mathrm{SO}_{2} 3(2,2) \rightarrow 2(1,1)$ & 208.7003 & $12^{\prime \prime}$ & 0.54 \\
$\mathrm{C}_{3} \mathrm{H}_{2} 2(1,2) \rightarrow 1(0,1)$ & 85.3389 & $29^{\prime \prime}$ & 0.80 \\
$\mathrm{C}_{3} \mathrm{H}_{2} 3(2,2) \rightarrow 2(1,1)$ & 155.5183 & $17^{\prime \prime}$ & 0.65 \\
$\mathrm{C}_{3} \mathrm{H}_{2} 3(3,0) \rightarrow 2(2,1)$ & 216.2787 & $17^{\prime \prime}$ & 0.65 \\
$\mathrm{C}_{3} \mathrm{H}_{2} 5(1,4) \rightarrow 4(2,3)$ & 217.9400 & $12^{\prime \prime}$ & 0.54 \\
$\mathrm{C}_{3} \mathrm{H}_{2} 5(2,4) \rightarrow 4(1,3)$ & 218.160 & $12^{\prime \prime}$ & 0.54 \\
$\mathrm{C}_{3} \mathrm{H}_{2} 6(0,6) \rightarrow 5(1,5)$ & 217.8220 & $12^{\prime \prime}$ & 0.54 \\
$\mathrm{C}_{3} \mathrm{H}_{2} 6(1,6) \rightarrow 5(0,5)$ & 217.8222 & $12^{\prime \prime}$ & 0.54 \\
\hline
\end{tabular}

\section{Observations}

The observations were carried out using the IRAM 30-m telescope in Pico de Veleta (Spain) during 24th-28th June 1998 and 17th-24th January, 2000. The multi-receiver capability of the telescope was used for these observations. The frequencies of the observed transitions and the telescope parameters are shown in Table 1. The backends used for the observations were an autocorrelator split in several parts, a $256 \times 100 \mathrm{kHz}$ and two $512 \times 1 \mathrm{MHz}$ filter-banks. Usually all the lines have been measured with two spectral resolutions, the $1 \mathrm{MHz}$ spectral resolution provided by the $512 \times 1 \mathrm{MHz}$ filter-banks and the higher spectral resolution of $78 \mathrm{kHz}-100 \mathrm{kHz}$ provided by the autocorrelator and the $100 \mathrm{kHz}$ filter bank. In Tables 2-6 we show the observational parameters derived from the high spectral resolution spectra. The $\mathrm{HOC}^{+} 1 \rightarrow 0$ and $\mathrm{HC}^{18} \mathrm{O}^{+} 1 \rightarrow 0$ lines were always observed simultaneously. In this way, we obtain a more accurate $\mathrm{HC}^{18} \mathrm{O}^{+} / \mathrm{HOC}^{+}$ratio avoiding the uncertainties due to calibration and pointing errors. The $\mathrm{C}_{3} \mathrm{H}_{2} 6(1,5) \rightarrow 5(0,5)$ [ortho] and $6(0,6) \rightarrow 5(1,5)$ [para] lines are blended. The Gaussian fits shown in the Tables are those obtained centering the spectrum at the frequency of the para line. Since the lines are blended, the areas are the sum of the areas of the ortho and para lines. The $\mathrm{SO}_{2} 3(2,2) \rightarrow 2(1,1)$ line at $208.7 \mathrm{GHz}$ was detected in the spectra obtained with the $512 \times 1 \mathrm{MHz}$ filter-banks when observing the $\mathrm{SO}^{+}$9/2 $\rightarrow 7 / 2$ lines.

\section{Results}

Significant differences are found between the PDRs (see Tables 2 to 6). The most abundant reactive molecular ion, $\mathrm{CO}^{+}$, has been detected towards all the targets. However, this is not the case for the other molecular ions. $\mathrm{HOC}^{+}$has not been detected towards NGC 7027. As we will discuss in the following sections, the upper limit to the emission of the $\mathrm{HOC}^{+}$line is significant and implies a different chemistry in this $\mathrm{PN}$ than in the other PDRs. The molecular ion $\mathrm{SO}^{+}$has been detected towards NGC 7023 and the Orion Bar. These constitute the first detections of $\mathrm{SO}^{+}$in PDRs. Similarly to the case of $\mathrm{HOC}^{+}, \mathrm{SO}^{+}$has 
Table 2. Observations of $\mathrm{HOC}^{+}$and $\mathrm{HC}^{18} \mathrm{O}^{+}$towards PDRs.

\begin{tabular}{|c|c|c|c|c|c|c|c|c|}
\hline Object & Line & $\begin{array}{c}\text { Area } \\
\left(\mathrm{K} \mathrm{km} \mathrm{s}^{-1}\right)\end{array}$ & $\begin{array}{c}V_{\mathrm{LSR}} \\
\left(\mathrm{km} \mathrm{s}^{-1}\right)\end{array}$ & $\begin{array}{c}\mathrm{HOC}^{+} \\
\Delta V \\
\left(\mathrm{~km} \mathrm{~s}^{-1}\right)\end{array}$ & $\begin{array}{l}T_{\text {rot }} \\
(\mathrm{K})\end{array}$ & $\begin{array}{l}N_{\mathrm{HOC}^{+}} \\
\left(\mathrm{cm}^{-2}\right)\end{array}$ & $\begin{array}{c}\mathrm{HC}^{18} \mathrm{O}^{+} \\
\text {Area } \\
\left(\mathrm{K} \mathrm{km} \mathrm{s}^{-1}\right)\end{array}$ & {$\left[\mathrm{HCO}^{+}\right] /\left[\mathrm{HOC}^{+}\right]$} \\
\hline \multicolumn{9}{|l|}{ NGC 7023} \\
\hline \multirow[t]{2}{*}{$(0,0)$} & $1 \rightarrow 0$ & $<0.019$ & & & $15^{a}$ & $<4.2 \times 10^{10}$ & $<0.026$ & \\
\hline & $3 \rightarrow 2$ & $<0.09$ & & & & & & \\
\hline \multirow[t]{2}{*}{$(-13,+16)$} & $1 \rightarrow 0$ & $<0.016$ & & & $15^{a}$ & $<3.5 \times 10^{10}$ & $<0.026$ & \\
\hline & $3 \rightarrow 2$ & $<0.03$ & & & & & & \\
\hline \multirow[t]{2}{*}{$(-25,+40)$} & $1 \rightarrow 0$ & $0.02(0.01)$ & $2.7(0.1)$ & $1.0(0.4)$ & $7-31^{b}$ & $2.7-7.6 \times 10^{10}$ & $<0.022$ & $50-120^{c}$ \\
\hline & $3 \rightarrow 2$ & $0.09(0.03)$ & $2.5(0.3)$ & $1.8(0.6)$ & & & & \\
\hline$(-30,+84)$ & $1 \rightarrow 0$ & $<0.15$ & & & $15^{a}$ & $<3.3 \times 10^{11}$ & & $>200^{c}$ \\
\hline \multicolumn{9}{|l|}{ Orion-Bar } \\
\hline$(+50,-50)$ & $1 \rightarrow 0$ & $<0.029$ & & & $15^{a}$ & $<6.4 \times 10^{10}$ & & \\
\hline$(+40,-40)$ & $1 \rightarrow 0$ & $0.11(0.02)$ & $9.7(0.2)$ & $2.1(0.5)$ & $15^{a}$ & $2.4 \times 10^{11}$ & & \\
\hline \multirow[t]{2}{*}{$(+20,-20)$} & $1 \rightarrow 0$ & $0.13(0.01)$ & $10.4(0.1)$ & $2.7(0.2)$ & $6-20^{b}$ & $1.7-3.5 \times 10^{11}$ & $0.08(0.01)$ & 400 \\
\hline & $3 \rightarrow 2$ & $0.37(0.04)$ & $10.7(0.1)$ & $2.4(0.3)$ & & & & \\
\hline \multirow[t]{2}{*}{$(+10,-10)$} & $1 \rightarrow 0$ & $0.09(0.01)$ & $10.3(0.1)$ & $2.7(0.3)$ & $7-30^{b}$ & $1.2-3.4 \times 10^{11}$ & $<0.030$ & $<217$ \\
\hline & $3 \rightarrow 2$ & $0.37(0.03)$ & $10.0(0.1)$ & $2.5(0.3)$ & & & & \\
\hline \multirow[t]{2}{*}{$(0,0)$} & $1 \rightarrow 0$ & $0.09(0.02)$ & $9.5(0.3)$ & $2.8(0.6)$ & $15^{a}$ & $2.0 \times 10^{11}$ & $<0.023$ & $<166$ \\
\hline & $3 \rightarrow 2$ & $<0.06$ & & & & & & \\
\hline
\end{tabular}

${ }^{a}$ Assumed rotation temperature.

${ }^{b}$ Lower and upper limits correspond to the rotation temperature assuming a point and extended source respectively.

${ }^{c}$ Derived from our limit to the $\mathrm{HOC}^{+} 1 \rightarrow 0$ emission and the $\mathrm{H}^{13} \mathrm{CO}^{+}$data reported by Fuente \& Martín-Pintado (1997).

Table 3. Observations of $\mathrm{CO}^{+}$towards PDRs.

\begin{tabular}{|c|c|c|c|c|c|c|c|c|}
\hline \multirow[b]{2}{*}{ Object } & \multirow[b]{2}{*}{ Offset } & \multicolumn{3}{|c|}{$\overline{\mathrm{CO}^{+} 2,5 / 2 \rightarrow 1,3 / 2}$} & \multicolumn{3}{|c|}{$\mathrm{CO}^{+} 2,1 / 2 \rightarrow 1,1 / 2$} & \multirow[b]{2}{*}{$\begin{array}{l}N_{\mathrm{CO}^{+}} \\
\left(\mathrm{cm}^{-2}\right)\end{array}$} \\
\hline & & $\begin{array}{c}\text { Area } \\
\left(\mathrm{K} \mathrm{km} \mathrm{s}^{-1}\right)\end{array}$ & $\begin{array}{c}V_{\mathrm{LSR}} \\
\left(\mathrm{km} \mathrm{s}^{-1}\right)\end{array}$ & $\begin{array}{c}\Delta V \\
\left(\mathrm{~km} \mathrm{~s}^{-1}\right)\end{array}$ & $\begin{array}{c}\text { Area } \\
\left(\mathrm{K} \mathrm{km} \mathrm{s}^{-1}\right)\end{array}$ & $\begin{array}{c}V_{\mathrm{LSR}} \\
\left(\mathrm{km} \mathrm{s}^{-1}\right)\end{array}$ & $\begin{array}{c}\Delta V \\
\left(\mathrm{~km} \mathrm{~s}^{-1}\right)\end{array}$ & \\
\hline \multirow[t]{4}{*}{ NGC 7023} & $(0,0)$ & $<0.04$ & & & $<0.04$ & & & $<6.4 \times 10^{10}$ \\
\hline & $(-13,+16)$ & $<0.04$ & & & $<0.04$ & & & $<6.4 \times 10^{10}$ \\
\hline & $(-25,+40)$ & $0.21(0.02)$ & $2.1(0.1)$ & $2.1(0.2)$ & $0.17(0.03)$ & $2.8(0.2)$ & $2.3(0.6)$ & $3.4 \times 10^{11}$ \\
\hline & $(-30,+84)$ & $<0.03$ & & & $<0.03$ & & & $<4.9 \times 10^{10}$ \\
\hline \multirow[t]{3}{*}{ Orion-Bar } & $(+20,-20)$ & $0.63(0.04)$ & $11.0(0.1)$ & $2.5(0.2)$ & $0.32(0.03)$ & $11.1(0.1)$ & $2.1(0.1)$ & $1.0 \times 10^{12}$ \\
\hline & $(+10,-10)$ & $1.45(0.05)$ & $10.8(0.1)$ & $3.1(0.1)$ & $0.82(0.03)$ & $10.8(0.1)$ & $2.8(0.3)$ & $2.4 \times 10^{12}$ \\
\hline & $(0,0)$ & $0.23(0.05)$ & $10.5(0.3)$ & $1.7(0.7)$ & $<0.17$ & & & $4.0 \times 10^{11}$ \\
\hline
\end{tabular}

not been detected towards NGC 7027. Strong $\mathrm{C}_{3} \mathrm{H}_{2}$ lines have been observed towards all the sources in our sample.

\subsection{NGC 7023}

We have observed the molecular ions, $\mathrm{CO}^{+}, \mathrm{HOC}^{+}, \mathrm{HC}^{18} \mathrm{O}^{+}$ and $\mathrm{SO}^{+}$, and the hydrocarbon compound $\mathrm{C}_{3} \mathrm{H}_{2}$ in four positions across the PDR: the star [offset $(0,0)$ ], an intermediate position between the star and the PDR-peak [offset $\left(-13^{\prime \prime},+16^{\prime \prime}\right)$ ], the PDR-peak [offset $\left(-25^{\prime \prime},+40^{\prime \prime}\right)$ ] and the molecular-peak [offset $\left.\left(-30^{\prime \prime},+84^{\prime \prime}\right)\right]$. We have marked these positions in Fig. 1. These positions constitute a strip that begins at the star position, crosses the PDR (PDR peak) and ends in the molecular cloud (molecular peak), sampling regions at different extinction from the star and consequently, with a different chemistry.

The molecular ions $\mathrm{CO}^{+}, \mathrm{HOC}^{+}$and $\mathrm{SO}^{+}$have only been detected towards the PDR-peak. In Fig. 3 we show the spectra of these molecular ions towards the PDR-peak and the molecular peak. For comparison we have also plotted the spectrum of the $\mathrm{HCO}^{+}$and $\mathrm{H}^{13} \mathrm{CO}^{+} 1 \rightarrow 0$ lines taken from Fuente \& Martín-Pintado (1997). While the intensity of the $\mathrm{H}^{13} \mathrm{CO}^{+}$line is two times stronger towards the molecular peak than towards the PDR-peak, the intensity of the $\mathrm{CO}^{+}$and $\mathrm{HOC}^{+}$lines is higher towards the PDR peak. As discussed in Fuente \& Martín-Pintado (1997), the hydrogen density towards the molecular-peak is $\sim 10^{5} \mathrm{~cm}^{-3}$, i.e., high enough to excite the $\mathrm{CO}^{+}, \mathrm{HOC}^{+}$and $\mathrm{SO}^{+}$lines. Then, the lack of detection of these ions towards the molecular-peak should be due to a gradient in their fractional abundances.

In this well-known nebula, we can use the kinematical information to study the structure of the PDR. The profile of $\mathrm{HCO}^{+}$shown in Fig. 3 is composed of a narrow component centered at the velocity of $2.4 \mathrm{kms}^{-1}$ and a wide plateau covering between 0 to $6 \mathrm{~km} \mathrm{~s}^{-1}$. Interferometric observations by Fuente et al. (1996) show that the $\mathrm{HCO}^{+}$emission arises in 
Table 4. Observations of $\mathrm{SO}^{+}$and $\mathrm{SO}_{2}$ towards PDRs.

\begin{tabular}{|c|c|c|c|c|c|c|c|c|c|}
\hline \multirow[b]{2}{*}{ Object } & \multirow[b]{2}{*}{ Offset } & \multicolumn{3}{|c|}{$\mathrm{SO}^{+}$9/2 $\rightarrow 7 / 2 \mathrm{e}$} & \multicolumn{3}{|c|}{$\mathrm{SO}_{2} 3_{2,2} \rightarrow 2_{1,1}$} & \multirow[b]{2}{*}{$\begin{array}{c}N_{\mathrm{SO}^{+}} \\
\left(\mathrm{cm}^{-2}\right)\end{array}$} & \multirow[b]{2}{*}{$\begin{array}{c}N_{\mathrm{SO}_{2}} \\
\left(\mathrm{~cm}^{-2}\right)\end{array}$} \\
\hline & & $\begin{array}{c}\text { Area } \\
\left(\mathrm{K} \mathrm{km} \mathrm{s}^{-1}\right)\end{array}$ & $\begin{array}{c}V_{\mathrm{LSR}} \\
\left(\mathrm{km} \mathrm{s}^{-1}\right)\end{array}$ & $\begin{array}{c}\Delta V \\
\left(\mathrm{~km} \mathrm{~s}^{-1}\right)\end{array}$ & $\begin{array}{c}\text { Area }^{a} \\
\left(\mathrm{~K} \mathrm{~km} \mathrm{~s}^{-1}\right)\end{array}$ & $\begin{array}{c}V_{\mathrm{LSR}} \\
\left(\mathrm{km} \mathrm{s}^{-1}\right)\end{array}$ & $\begin{array}{c}\Delta V \\
\left(\mathrm{~km} \mathrm{~s}^{-1}\right)\end{array}$ & & \\
\hline \multirow[t]{4}{*}{ NGC 7023} & $(0,0)$ & $<0.05^{b}$ & & & $<0.05$ & & & $<5 \times 10^{11}$ & $<1.5 \times 10^{12}$ \\
\hline & $(-13,+16)$ & $<0.05^{b}$ & & & $<0.05$ & & & $<5 \times 10^{11}$ & $<1.5 \times 10^{12}$ \\
\hline & $(-25,+40)$ & $0.06(0.01)$ & $2.4(0.1)$ & $0.8(0.2)$ & $<0.05$ & & & $6 \times 10^{11}$ & $<1.5 \times 10^{12}$ \\
\hline & $(-30,+84)$ & $<0.05^{b}$ & & & $<0.04$ & & & $<5 \times 10^{11}$ & $<1.2 \times 10^{12}$ \\
\hline \multirow[t]{3}{*}{ Orion-Bar } & $(+20,-20)$ & $0.84(0.03)$ & $10.5(0.1)$ & $2.3(0.1)$ & $0.67(0.02)$ & $9.8(0.1)$ & $2(1)$ & $8.4 \times 10^{12}$ & $2.0 \times 10^{13}$ \\
\hline & $(+10,-10)$ & $0.44(0.04)$ & $10.3(0.1)$ & $2.5(0.3)$ & $0.12(0.02)$ & $10.0(0.1)$ & $1(1)$ & $4.4 \times 10^{12}$ & $3.6 \times 10^{12}$ \\
\hline & $(0,0)$ & $0.12(0.02)$ & $10.0(0.1)$ & $1.2(0.2)$ & $<0.04$ & & & $1.2 \times 10^{12}$ & $<1.2 \times 10^{12}$ \\
\hline
\end{tabular}

${ }^{a} \mathrm{SO}_{2}$ has been observed with a spectral resolution of $1 \mathrm{MHz}\left(1.4 \mathrm{~km} \mathrm{~s}^{-1}\right)$.

${ }^{b}$ 3- $\sigma$ area upper limit with $\Delta v=0.9 \mathrm{~km} \mathrm{~s}^{-1}$.

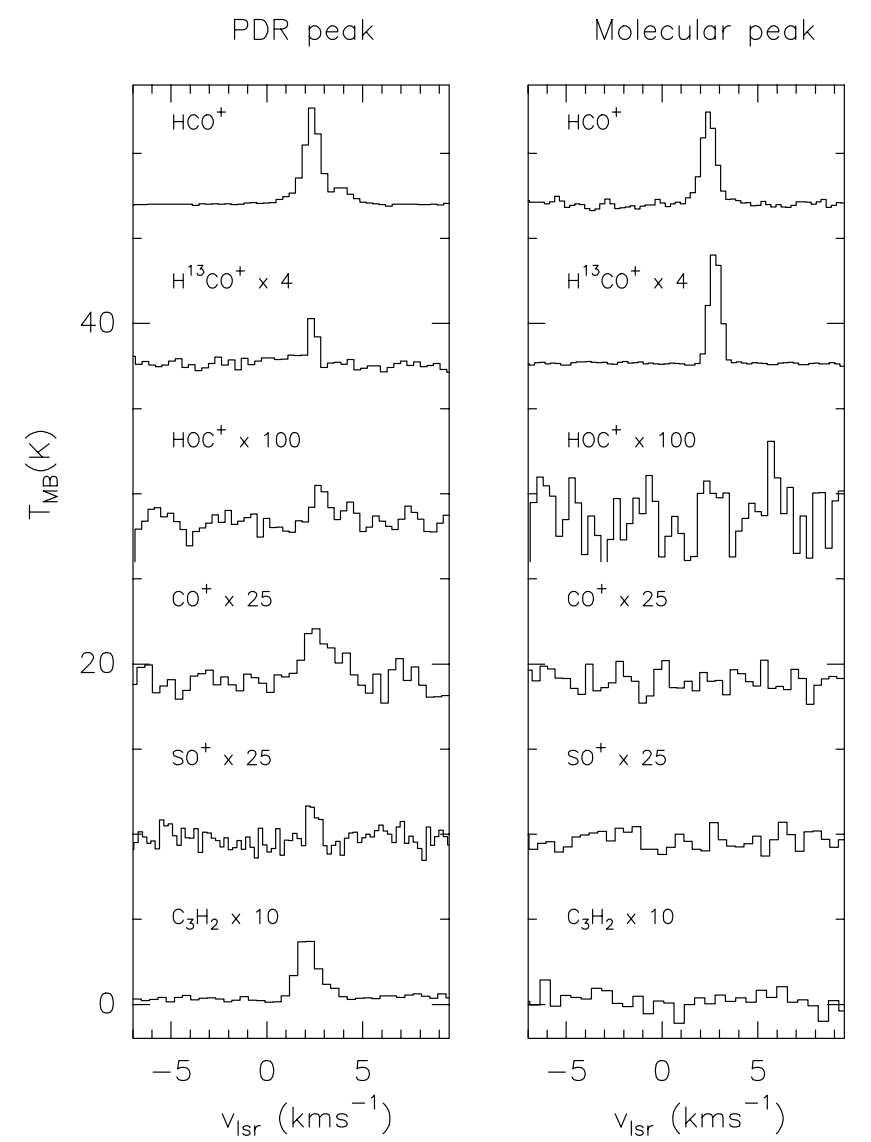

Fig. 3. Spectra of the $\mathrm{HCO}^{+} 1 \rightarrow 0, \mathrm{H}^{13} \mathrm{CO}^{+} 1 \rightarrow 0, \mathrm{HOC}^{+} 1 \rightarrow 0, \mathrm{CO}^{+}$ $2,5 / 2 \rightarrow 1,3 / 2, \mathrm{SO}^{+} 9 / 2 \rightarrow 7 / 2$ (e) and $\mathrm{C}_{3} \mathrm{H}_{2} 6(1,6) \rightarrow 5(0,5)$ towards the PDR-peak [offset $\left(-25^{\prime \prime}, 40^{\prime \prime}\right)$ relative to the star position] and the molecular-peak [offset $\left(-30^{\prime \prime}, 84^{\prime \prime}\right)$ relative to the star position] in NGC 7023.

several high-density filaments located at the interface between the atomic and molecular gas. These filaments have slightly different velocities ranging between $1 \mathrm{~km} \mathrm{~s}^{-1}$ and $6 \mathrm{~km} \mathrm{~s}^{-1}$ producing the wide wings in the single-dish spectrum. While the filament at $2.4 \mathrm{~km} \mathrm{~s}^{-1}$ is already embedded in the molecular cloud, the filaments at more positive velocities $\left(v>3.8 \mathrm{~km} \mathrm{~s}^{-1}\right)$ are embedded in the atomic gas. The filament at $2.4 \mathrm{~km} \mathrm{~s}^{-1}$ is the only one detected in the $\mathrm{H}^{13} \mathrm{CO}^{+}$line with a linewidth of $0.7 \mathrm{~km} \mathrm{~s}^{-1}$. This is also the case for most molecular lines (see the CS lines in Fuente et al. 1997) which present linewidths of $<1 \mathrm{~km} \mathrm{~s}^{-1}$. However, the linewidths of the $\mathrm{CO}^{+}$and $\mathrm{HOC}^{+}$ lines are clearly broader. Comparing the profile of the $\mathrm{HCO}^{+}$ $1 \rightarrow 0$ line with those of $\mathrm{CO}^{+}$and $\mathrm{HOC}^{+}$, it seems clear that the emission of these reactive ions arise mainly in the high density filaments immersed in the atomic phase.

The lines of the hydrocarbon radical $\mathrm{C}_{3} \mathrm{H}_{2}$ also present broad linewidths $\left(>1 \mathrm{~km} \mathrm{~s}^{-1}\right)$. Several $\mathrm{C}_{3} \mathrm{H}_{2}$ transitions with different excitation conditions have been observed towards NGC 7023. The high excitation $6(0,6) \rightarrow 5(1,5)$ and $6(1,6) \rightarrow 5(0,5)$ lines have only been detected towards the PDRpeak. The comparison between the spectra towards the PDRpeak and the molecular-peak suggests a different $\mathrm{C}_{3} \mathrm{H}_{2}$ abundance towards these two positions. Moreover, the linewidth of this line is broader than that of the $\mathrm{H}^{13} \mathrm{CO}^{+}$line suggesting that this species arises in the high density filaments in the interface between the atomic and molecular phase.

The low excitation $\mathrm{C}_{3} \mathrm{H}_{2} 2(1,2) \rightarrow 1(0,1)$ line has also been detected towards the star position. Low total column density, $\mathrm{N}\left(\mathrm{H}_{2}\right) \sim 2 \times 10^{21} \mathrm{~cm}^{-2}$, and low hydrogen density, $n \sim$ $10^{3} \mathrm{~cm}^{-3}$, have been measured towards this position (Fuente et al. 1998; Gerin et al. 1998). The detection of $\mathrm{C}_{3} \mathrm{H}_{2}$ reveals that this molecule is also abundant in this diffuse gas.

Finally, the case of $\mathrm{SO}^{+}$is slightly different. Similarly to $\mathrm{CO}^{+}$and $\mathrm{HOC}^{+}, \mathrm{SO}^{+}$has only been detected towards the PDRpeak. However, the $\mathrm{SO}^{+}$lines are very narrow with a linewidth similar to those of the $\mathrm{H}^{13} \mathrm{CO}^{+}$and $\mathrm{CS}$ lines.

\subsection{The Orion Bar}

We have observed a strip crossing the ionization front (IF) of the Orion $\mathrm{Bar}$ in $\mathrm{CO}^{+}, \mathrm{HOC}^{+}, \mathrm{HC}^{18} \mathrm{O}^{+}, \mathrm{SO}^{+}$and $\mathrm{C}_{3} \mathrm{H}_{2}$ lines (see Fig. 2). The number of observed positions along the strip is different for each species. The observed positions and observational parameters for all the molecular lines are shown in Tables 2 to 5 . The offset $(0,0)$ is the position of the IF $\left(\operatorname{RA}(2000)=05^{\mathrm{h}} 35^{\mathrm{m}} 20^{\mathrm{s}} \cdot 1 \operatorname{Dec}(2000)=-05^{\circ} 25^{\prime} 07^{\prime \prime}\right.$.0 $)$. The offset $\left(+20^{\prime \prime},-20^{\prime \prime}\right)$ is the maximum in $\mathrm{H}_{2}$ column density (Jansen et al. 1995; Fuente et al. 1996), and hereafter we will refer to 
Table 5. Observations of $\mathrm{C}_{3} \mathrm{H}_{2}$.

\begin{tabular}{|c|c|c|c|c|c|c|c|}
\hline Object & Offset & Line & $\begin{array}{c}\text { Area } \\
\left(\mathrm{K} \mathrm{km} \mathrm{s}^{-1}\right)\end{array}$ & $\begin{array}{c}V_{\mathrm{LSR}} \\
\left(\mathrm{km} \mathrm{s}^{-1}\right)\end{array}$ & $\begin{array}{c}\Delta V \\
\left(\mathrm{~km} \mathrm{~s}^{-1}\right)\end{array}$ & $\begin{array}{l}T_{\mathrm{ex}} \\
(\mathrm{K})\end{array}$ & $\begin{array}{c}N_{\mathrm{C}_{3} \mathrm{H}_{2}} \\
\left(10^{12} \mathrm{~cm}^{-2}\right)\end{array}$ \\
\hline \multirow[t]{13}{*}{ NGC 7023} & $(0,0)$ & $2(1,2) \rightarrow 1(0,1)$ & $0.05(0.02)$ & $1.2(0.5)$ & $3.2(0.9)$ & $15^{a}$ & 0.42 \\
\hline & & $3(2,2) \rightarrow 2(1,1)$ & $<0.03$ & & & & \\
\hline & & $5(1,4) \rightarrow 4(2,3)$ & $<0.04$ & & & & \\
\hline & & $5(2,4) \rightarrow 4(1,3)$ & $<0.04$ & & & & \\
\hline & & $6(0,6) \rightarrow 5(1,5)$ & $<0.04$ & & & & \\
\hline & $(-13,+16)$ & $5(1,4) \rightarrow 4(2,3)$ & $<0.02$ & & & $15^{a}$ & $<0.2$ \\
\hline & & $5(2,4) \rightarrow 4(1,3)$ & $<0.10$ & & & & \\
\hline & & $6(0,6) \rightarrow 5(1,5)$ & $<0.14$ & & & & \\
\hline & $(-25,+40)$ & $2(1,2) \rightarrow 1(0,1)$ & $0.25(0.01)$ & $2.6(0.1)$ & $1.1(0.1)$ & $8-15$ & $1.2-2.1$ \\
\hline & & $5(1,4) \rightarrow 4(2,3)$ & $0.15(0.01)$ & $2.2(0.1)$ & $0.9(0.1)$ & & \\
\hline & & $5(2,4) \rightarrow 4(1,3)$ & $0.06(0.01)$ & $2.2(0.1)$ & $1.0(0.3)$ & & \\
\hline & & $6(0,6) \rightarrow 5(1,5)$ & $0.42(0.02)$ & $2.1(0.1)$ & $1.2(0.1)$ & & \\
\hline & $(-30,+80)$ & $6(0,6) \rightarrow 5(1,5)$ & $<0.10$ & & & $15^{a}$ & $<0.8$ \\
\hline \multirow[t]{29}{*}{ Orion-Bar } & $(+50,-50)$ & $6(0,6) \rightarrow 5(1,5)$ & $0.28(0.07)$ & $9.8(0.2)$ & $1.6(0.6)$ & $15^{a}$ & 1.6 \\
\hline & $(+40,-40)$ & $5(1,4) \rightarrow 4(2,3)$ & $0.40(0.10)$ & & $3.9(1.2)$ & $15^{a}$ & 3.0 \\
\hline & & $6(0,6) \rightarrow 5(1,5)$ & $0.51(0.1)$ & $10.1(0.3)$ & $2.7(0.7)$ & & \\
\hline & $(+30,-30)$ & $5(1,4) \rightarrow 4(2,3)$ & $0.62(0.12)$ & & $2.7(0.6)$ & $15^{a}$ & 8.3 \\
\hline & & $6(0,6) \rightarrow 5(1,5)$ & $1.06(0.05)$ & $10.6(0.1)$ & $2.2(0.1)$ & & \\
\hline & $(+20,-20)$ & $2(1,2) \rightarrow 1(0,1)$ & $2.2(0.1)$ & $10.9(0.0)$ & $2.6(0.1)$ & 13 & 14 \\
\hline & & $3(2,2) \rightarrow 2(1,1)$ & $0.50(0.02)$ & $10.1(0.1)$ & $2.2(0.1)$ & & \\
\hline & & $5(1,4) \rightarrow 4(2,3)$ & $1.4(0.1)$ & $11.2(0.1)$ & $2.5(0.2)$ & & \\
\hline & & $5(2,4) \rightarrow 4(1,3)$ & $0.4(0.5)$ & & $2(3)$ & & \\
\hline & & $6(0,6) \rightarrow 5(1,5)$ & $1.73(0.08)$ & $10.6(0.0)$ & $2.7(0.1)$ & & \\
\hline & $(+10,-10)$ & $2(1,2) \rightarrow 1(0,1)$ & $1.7(0.1)$ & $11.0(0.1)$ & $2.5(0.1)$ & 15 & 13 \\
\hline & & $3(2,2) \rightarrow 2(1,1)$ & $0.7(0.1)$ & $10.0(0.1)$ & $2.5(0.3)$ & & \\
\hline & & $3(3,0) \rightarrow 2(2,1)$ & $0.9(0.1)$ & $11.2(0.1)$ & $1.9(0.2)$ & & \\
\hline & & $5(1,4) \rightarrow 4(2,3)$ & $1.3(0.2)$ & & $3.1(0.9)$ & & \\
\hline & & $5(2,4) \rightarrow 4(1,3)$ & $0.4(0.1)$ & $10.6(0.3)$ & $2.9(0.6)$ & & \\
\hline & & $6(0,6) \rightarrow 5(1,5)$ & $2.35(0.04)$ & $10.7(0.1)$ & $2.3(0.1)$ & & \\
\hline & $(0,0)$ & $2(1,2) \rightarrow 1(0,1)$ & $1.20(0.03)$ & $10.7(0.1)$ & $2.6(0.1)$ & 13 & 9 \\
\hline & & $3(2,2) \rightarrow 2(1,1)$ & $0.40(0.02)$ & $9.8(0.1)$ & $3.3(0.1)$ & & \\
\hline & & $5(1,4) \rightarrow 4(2,3)$ & $0.89(0.06)$ & $10.5(0.1)$ & $3.2(0.3)$ & & \\
\hline & & $5(2,4) \rightarrow 4(1,3)$ & $0.19(0.02)$ & $10.4(0.1)$ & $1.9(0.3)$ & & \\
\hline & & $6(0,6) \rightarrow 5(1,5)$ & $1.12(0.03)$ & $10.1(0.1)$ & $2.5(0.1)$ & & \\
\hline & $(-10,+10)$ & $2(1,2) \rightarrow 1(0,1)$ & $1.04(0.03)$ & $10.4(0.1)$ & $3.1(0.1)$ & 12 & 7 \\
\hline & & $5(1,4) \rightarrow 4(2,3)$ & $0.73(0.04)$ & $10.3(0.1)$ & $5.6(0.4)$ & & \\
\hline & & $5(2,4) \rightarrow 4(1,3)$ & $0.09(0.03)$ & $9.1(0.3)$ & $1.9(0.8)$ & & \\
\hline & & $6(0,6) \rightarrow 5(1,5)$ & $0.96(0.06)$ & & $3.6(0.2)$ & & \\
\hline & $(-20,+20)$ & $2(1,2) \rightarrow 1(0,1)$ & $1.09(0.03)$ & $10.1(0.1)$ & $3.2(0.1)$ & 12 & 8 \\
\hline & & $5(1,4) \rightarrow 4(2,3)$ & $0.6(0.1)$ & $8.7(0.1)$ & $3.4(0.3)$ & & \\
\hline & & $5(2,4) \rightarrow 4(1,3)$ & $0.2(0.1)$ & & $2(1)$ & & \\
\hline & & $6(0,6) \rightarrow 5(1,5)$ & $0.9(0.1)$ & $8.8(0.1)$ & $3.0(0.3)$ & & \\
\hline
\end{tabular}

${ }^{a}$ Assumed rotation temperature.

it as the molecular-peak. Spectra of the observed lines towards the IF, the molecular peak and an intermediate position [offset $(+10,-10)]$ are shown in Fig. 4. For an easier comparison, we also show in Fig. 5 the integrated intensities of the $\mathrm{HOC}^{+}$ $1 \rightarrow 0, \mathrm{CO}^{+} 2,5 / 2 \rightarrow 1,3 / 2, \mathrm{SO}^{+}$9/2 $\rightarrow 7 / 2 \mathrm{e}, \mathrm{C}_{3} \mathrm{H}_{2} 6(1,6) \rightarrow 5(0,5)$ and $\mathrm{HC}^{18} \mathrm{O}^{+} 1 \rightarrow 0$ lines normalized to 1 . The $\mathrm{CO}^{+}, \mathrm{SO}^{+}$and the $\mathrm{C}_{3} \mathrm{H}_{2} \quad 6(1,6) \rightarrow 5(0,5)$ lines have similar frequencies and have been observed with a beam of $\sim 12^{\prime \prime}$ (see Table 1 ). The comparison between them is straightforward. The $\mathrm{HOC}^{+}$and $\mathrm{HC}^{18} \mathrm{O}^{+}$lines have been observed with a larger beam of $\sim 29^{\prime \prime}$.

There are significant differences between the spatial distribution of the different species (see Fig. 5). The $\mathrm{HC}^{18} \mathrm{O}^{+}$and $\mathrm{SO}_{2}$ lines have only been detected towards the molecular peak. However, the $\mathrm{CO}^{+}$and all the $\mathrm{C}_{3} \mathrm{H}_{2}$ lines peak closer to the IF, at the position $\left(+10^{\prime \prime},-10^{\prime \prime}\right)$. The excitation conditions are quite uniform across the Orion Bar (Hogerheijde et al. 1995; 
Table 6. Observational results in NGC 7027.

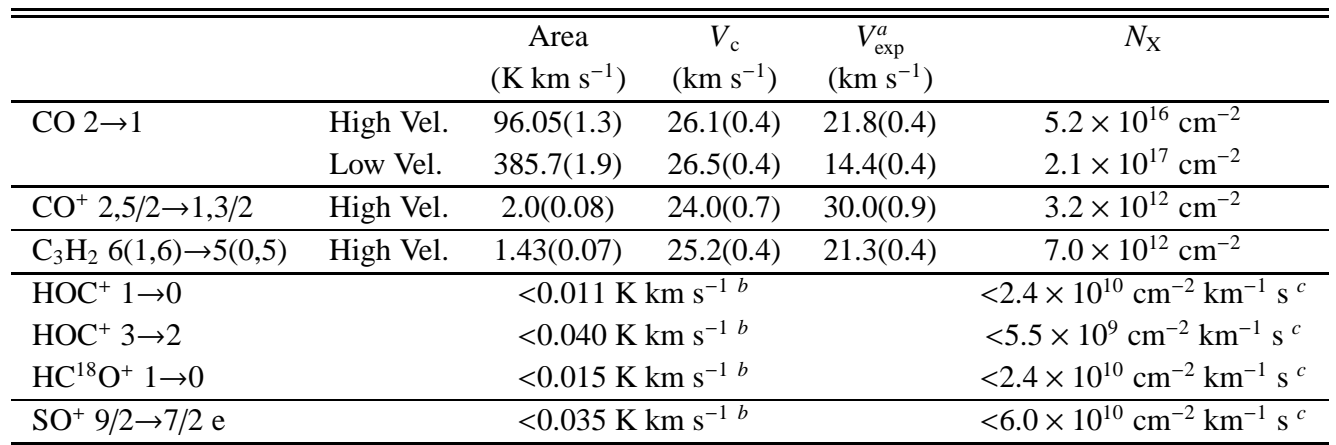

${ }^{a}$ Lines have been fitted with a typical shell profile. The fitted parameters are: Area, Position $\left(V_{\mathrm{c}}\right)$ and full width at zero level $\left(2 \times V_{\mathrm{exp}}\right)$.

${ }^{b}$ 3-sigma upper limit to the integrated intensity in a velocity interval of $1 \mathrm{~km} \mathrm{~s}^{-1}$.

${ }^{c} 3$-sigma upper limit for $N / \Delta v$ assuming a source size of $13^{\prime \prime}$ and an expansion velocity of $20 \mathrm{~km} \mathrm{~s}^{-1}\left(\Delta v \sim 40 \mathrm{~km} \mathrm{~s}^{-1}\right)$.

Fuente et al. 1996b). This spatial distribution strongly suggests that the fractional abundances of $\mathrm{CO}^{+}$and $\mathrm{C}_{3} \mathrm{H}_{2}$ are enhanced close to the IF. The molecular ions $\mathrm{HOC}^{+}$and $\mathrm{SO}^{+}$represent an intermediate case. Although the abundances of both molecules also seem to increase toward the IF (note in Fig. 5 that the $\mathrm{HOC}^{+} / \mathrm{HC}^{18} \mathrm{O}^{+}$and $\mathrm{SO}^{+} / \mathrm{HC}^{18} \mathrm{O}^{+}$ratio increase towards the IF), their emission peaks at the molecular peak.

\subsection{NGC 7027}

Intense emission has been detected in the $\mathrm{CO}^{+}$and $\mathrm{C}_{3} \mathrm{H}_{2}$ lines. This is in contrast with the non-detection of $\mathrm{HOC}^{+}$and $\mathrm{SO}^{+}$. In Fig. 4 we show the spectra of the $\mathrm{C}_{3} \mathrm{H}_{2} 6(1,6) \rightarrow 5(0,5), \mathrm{CO}^{+}$ $2,5 / 2 \rightarrow 1,3 / 2$, and ${ }^{12} \mathrm{CO} 2 \rightarrow 1$ lines. The linewidths of the $\mathrm{CO}^{+}$ $2,5 / 2 \rightarrow 1,3 / 2$ and the $\mathrm{C}_{3} \mathrm{H}_{2} \quad 6(1,6) \rightarrow 5(0,5)$ lines are broader than that of the $\mathrm{CO}$ line suggesting that the emission arises in a high-velocity layer of the envelope. Fitting the $\mathrm{C}_{3} \mathrm{H}_{2}$ spectra with a shell model we measure an expansion velocity of $v_{\mathrm{e}} \sim 21.5 \mathrm{~km} \mathrm{~s}^{-1}$ for this layer. An even higher expansion velocity of $v_{\mathrm{e}} \sim 30 \mathrm{~km} \mathrm{~s}^{-1}$ is obtained from the $\mathrm{CO}^{+}$spectrum. The spectrum of the ${ }^{12} \mathrm{CO}$ line reveals the existence of two components, a low velocity component with an expansion velocity of $\sim 14.4 \mathrm{~km} \mathrm{~s}^{-1}$ from which arises the bulk of the ${ }^{12} \mathrm{CO}$ emission and a higher velocity shell with an expansion velocity of $\sim 21.5 \mathrm{~km} \mathrm{~s}^{-1}$ which emits very weakly in CO. The $\mathrm{CO}^{+}$and $\mathrm{C}_{3} \mathrm{H}_{2}$ emission arise mainly in the high velocity shell (see Table 6). The intense emission of $\mathrm{CO}^{+}$and $\mathrm{C}_{3} \mathrm{H}_{2}$ in this high velocity shell is consistent with this gas being the innermost layer of the neutral envelope which is being accelerated by the ionized gas (Hasegawa \& Kwok 2001). In this layer, the chemistry of the gas is expected to be stongly affected by the stellar UV radiation.

\section{Column density and fractional abundance estimates}

The method used to estimate column densities and the assumptions we have made are explained in Appendix A. The derived column densities are shown in Tables 2 to 6. Fractional abundances are shown in Table 7 . When possible we have carried out excitation calculations in order to obtain accurate column densities. In some cases we have detected only one transition of the studied species and we need to assume a rotation temperature to derive the molecular column density. This is always the case for the $\mathrm{CO}^{+}, \mathrm{SO}^{+}$and $\mathrm{SO}_{2}$ lines. In these cases we have assumed a rotation temperature based on previous observations of lines with similar excitation conditions. The excitation calculations carried out for the $\mathrm{C}_{3} \mathrm{H}_{2}$ and $\mathrm{HOC}^{+}$lines in NGC 7023 and the Orion Bar (this paper) and for the $\mathrm{H}^{13} \mathrm{CO}^{+}$lines in NGC 7023 (Fuente \& Martín-Pintado 1997) give rotation temperatures between $6 \mathrm{~K}$ and $20 \mathrm{~K}$ in these PDRs. Thus, we have adopted a rotation temperature of $10 \mathrm{~K}$ for the $\mathrm{CO}^{+}$, $\mathrm{SO}^{+}$and $\mathrm{SO}_{2}$ lines in these sources which should be accurate within a factor of 2. Hasegawa \& Kwok (2001) derived excitation temperatures $\sim 20 \mathrm{~K}$ for the $\mathrm{HCO}^{+}, \mathrm{HCN}$ and $\mathrm{CN}$ lines in NGC 7027. A lower rotation temperature of $\sim 11 \mathrm{~K}$ was derived for the $\mathrm{CO}^{+}$lines. We have adopted $T_{\mathrm{r}}=20 \mathrm{~K}$ for the $\mathrm{HOC}^{+}$ and $\mathrm{C}_{3} \mathrm{H}_{2}$ lines and $T_{\mathrm{r}}=10 \mathrm{~K}$ for the $\mathrm{CO}^{+}$and $\mathrm{SO}^{+}$lines in this PN, which should also be accurate within a factor of 2 . Since the assumed rotation temperatures are accurate within a factor of 2, the uncertainties in the derived column densities because of the unknown excitation conditions are also of a factor $\sim 2$. Opacity effects are not important in the emission of these low abundance species. Therefore, in our discussion we will consider that variations of the molecular abundance ratios and fractional abundances by more than a factor of $\sim 4$ are significant.

\subsection{NGC 7023}

The molecular ion $\mathrm{HOC}^{+}$has only been detected towards the PDR-peak. For this position we have assumed that the size of the source matches that of the beam at $268 \mathrm{GHz}$ and estimated a rotation temperature of $\approx 30 \mathrm{~K}$. With this rotation temperature, the column density of $\mathrm{HOC}^{+}$is $\sim 8 \times 10^{10} \mathrm{~cm}^{-2}$. In order to estimate the uncertainty due to the beam filling factor, we have calculated the rotation temperature assuming an extended source and obtained a lower limit to the rotation temperature and column density of $7 \mathrm{~K}$ and of $\sim 3 \times 10^{10} \mathrm{~cm}^{-2}$ respectively. Assuming a total $\mathrm{H}_{2}$ density of $1.1 \times 10^{22} \mathrm{~cm}^{-2}$ (Fuente \& Martín-Pintado 1997) we derive an $\mathrm{HOC}^{+}$fractional abundance of $\sim 4 \times 10^{-12}-7 \times 10^{-12}$ 

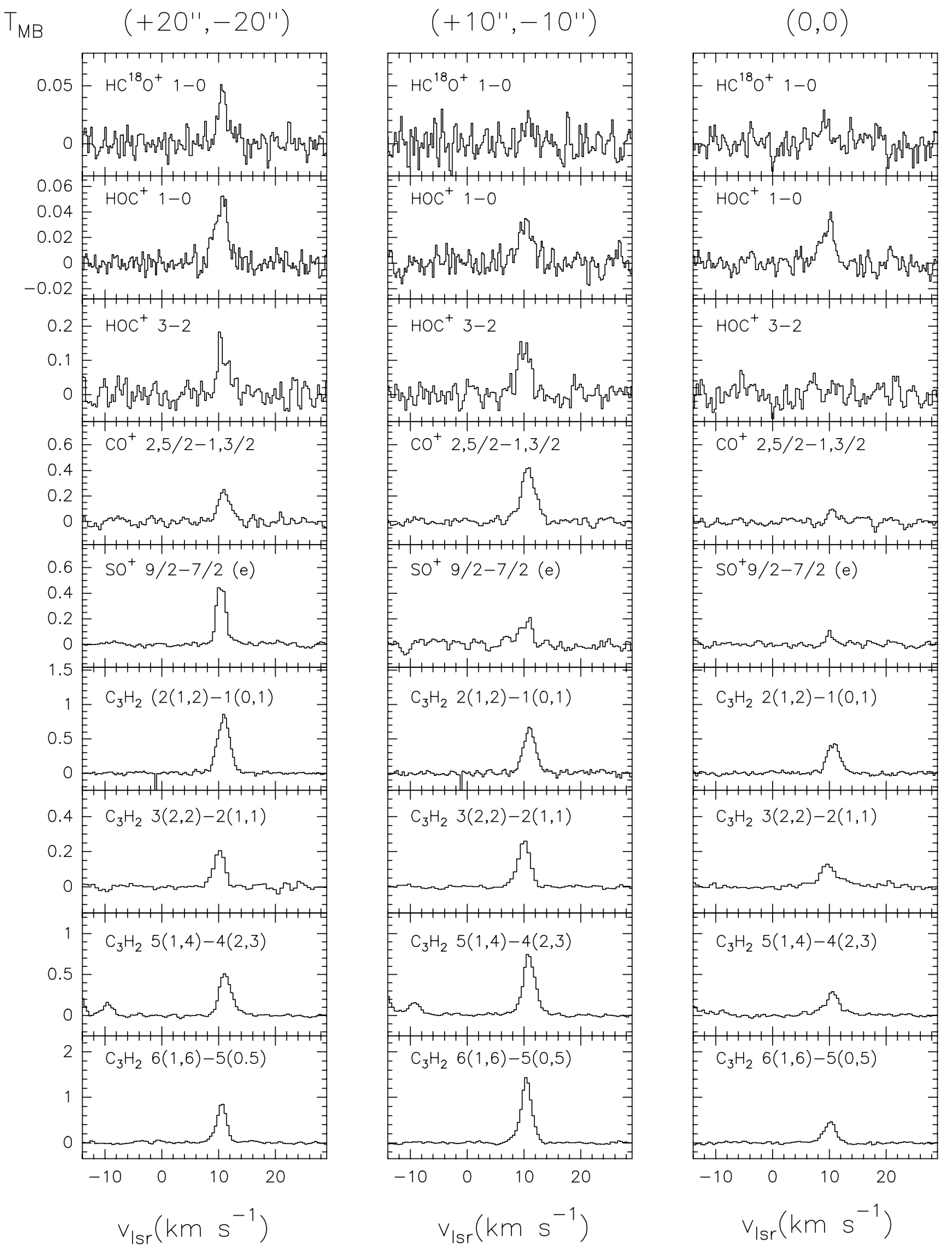

Fig. 4. Spectra of the observed molecular lines towards the position $\left(20^{\prime \prime},-20^{\prime \prime}\right),\left(10^{\prime \prime},-10^{\prime \prime}\right)$ and $(0,0)$ of the Orion Bar. The offset $(0,0)$ corresponds to the position of the IF. The offset $\left(20^{\prime \prime},-20^{\prime \prime}\right)$ corresponds to the maximum of molecular hydrogen column density.

depending on the assumed source size. We have not detected $\mathrm{HC}^{18} \mathrm{O}^{+}$towards the PDR-peak, thus we use our previous $\mathrm{HCO}^{+}$and $\mathrm{H}^{13} \mathrm{CO}^{+}$data to calculate the $\left[\mathrm{HCO}^{+}\right] /\left[\mathrm{HOC}^{+}\right]$ratio. Fuente \& Martín-Pintado (1997) estimated an $\mathrm{HCO}^{+}$column density of $4.5 \times 10^{12} \mathrm{~cm}^{-2}$ towards the PDR-peak, which implies an $\left[\mathrm{HCO}^{+}\right] /\left[\mathrm{HOC}^{+}\right]$ratio of $50-120$, which is the lowest ratio found thus far. We have not detected $\mathrm{HOC}^{+}$towards the molecular peak. Assuming a rotation temperature of $15 \mathrm{~K}$, we derive a $3-\sigma$ upper limit to the $\mathrm{HOC}^{+}$column density of $<4 \times 10^{11} \mathrm{~cm}^{-2}$. Taking into account the data reported by Fuente \& Martín-Pintado (1997), we obtain an $\mathrm{HOC}^{+}$fractional abundance $<2 \times 10^{-12}$ and an $\left[\mathrm{HCO}^{+}\right] /\left[\mathrm{HOC}^{+}\right]$ratio $>$ 200 towards this position, i.e., at least a factor of 2 larger than towards the PDR-peak.

The molecular ion $\mathrm{CO}^{+}$has only been detected towards the PDR-peak. Observations of $\mathrm{CO}^{+}$in NGC 7023 were previously reported by Fuente \& Martín-Pintado (1997). Our new data are in total agreement with previous ones. We have estimated a $\mathrm{CO}^{+}$column density of $3.4 \times 10^{11} \mathrm{~cm}^{-2}$ towards the PDR-peak. The fractional abundance of $\mathrm{CO}^{+}$is $3 \times 10^{-11}$, an order of 
Table 7. Fractional abundances in PDRs.

\begin{tabular}{|c|c|c|c|c|c|c|c|}
\hline & & $\mathrm{HOC}^{+}$ & $\mathrm{CO}^{+}$ & $\mathrm{SO}^{+}$ & $\mathrm{C}_{3} \mathrm{H}_{2}$ & $\mathrm{HCO}^{+} / \mathrm{HOC}^{+}$ & $\mathrm{SO}^{+} / \mathrm{SO}_{2}$ \\
\hline \multirow[t]{3}{*}{ NGC 7023} & PDR-peak & $7 \times 10^{-12}$ & $3 \times 10^{-11}$ & $5 \times 10^{-11}$ & $2 \times 10^{-10}$ & $50-120$ & $>0.4$ \\
\hline & $\left(-25^{\prime \prime},+40^{\prime \prime}\right)$ & & & & & & \\
\hline & $\begin{array}{l}\text { Mol-peak } \\
\left(-30^{\prime \prime},+84^{\prime \prime}\right)\end{array}$ & $<2 \times 10^{-12}$ & $<3 \times 10^{-13}$ & $<3 \times 10^{-12}$ & $<3 \times 10^{-12}$ & $>200$ & \\
\hline \multirow[t]{2}{*}{ Orion Bar } & $\begin{array}{l}\text { IF } \\
(0,0)\end{array}$ & $3 \times 10^{-11}$ & $6 \times 10^{-11}$ & $1 \times 10^{-10}$ & $2 \times 10^{-9}$ & $<166$ & $<1$ \\
\hline & $\begin{array}{l}\text { Mol-peak } \\
\left(+20^{\prime \prime},-20^{\prime \prime}\right)\end{array}$ & $5 \times 10^{-12}$ & $1 \times 10^{-11}$ & $1 \times 10^{-10}$ & $2 \times 10^{-10}$ & 400 & 0.4 \\
\hline NGC 7027 & & & $5 \times 10^{-9}$ & & $1 \times 10^{-8}$ & $>450$ & \\
\hline
\end{tabular}

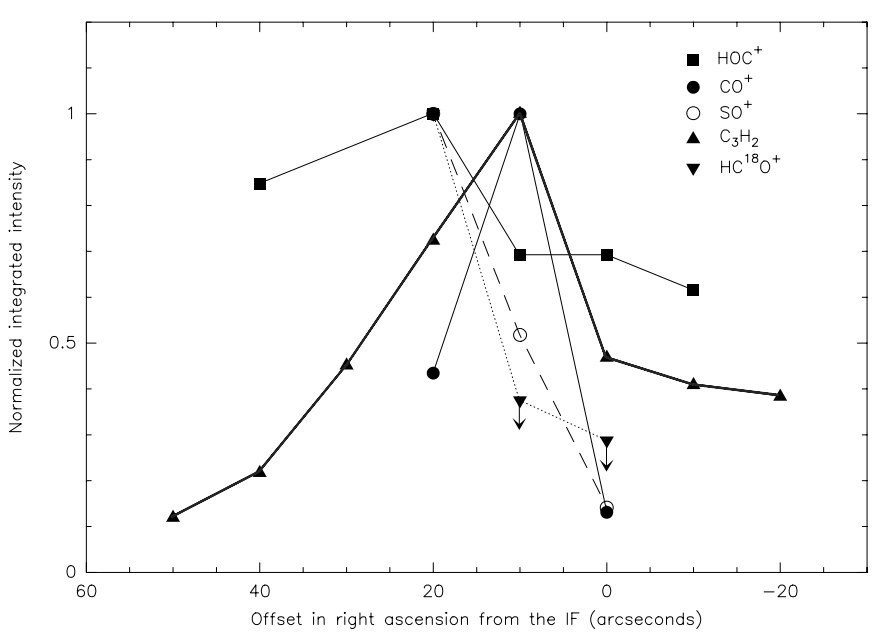

Fig. 5. Normalized integrated intensity of the $\mathrm{HOC}^{+} 1 \rightarrow 0, \mathrm{CO}^{+}$ $2,5 / 2 \rightarrow 1,3 / 2, \mathrm{SO}^{+} 9 / 2 \rightarrow 7 / 2(\mathrm{e}), \mathrm{C}_{3} \mathrm{H}_{2} \quad 6(1,6) \rightarrow 5(0,5)$ and $\mathrm{HC}^{18} \mathrm{O}^{+}$ $1 \rightarrow 0$ lines as a function of the offset in right ascension relative to the posisition of the IF for the strip observed towards the Orion Bar.

magnitude larger than that of $\mathrm{HOC}^{+}$. We have not detected $\mathrm{CO}^{+}$towards the molecular peak with a fractional abundance at least 100 times lower than towards the molecular peak (Fuente \& Martín-Pintado 1997)

Similarly to $\mathrm{CO}^{+}, \mathrm{SO}^{+}$has only been detected towards the PDR-peak. We have estimated a SO${ }^{+}$abundance of $5.4 \times 10^{-11}$ towards this position. This abundance is similar to those found by Turner (1994) towards a sample of star forming regions. The fractional abundance of $\mathrm{SO}^{+}$decreases inside the molecular cloud. Our upper limit for the $\mathrm{SO}^{+}$abundance towards the molecular peak is $<3 \times 10^{-12}$, i.e., a factor of 10 lower than towards the PDR-peak.

As discussed in Sect. 4.1, the profile of the $\mathrm{SO}^{+}$lines is very narrow in contrast with those of the other reactive ions. Comparing the profiles of the $\mathrm{SO}^{+}$and $\mathrm{CO}^{+}$lines, $\mathrm{SO}^{+}$seems to arise only in the filament at $2.4 \mathrm{~km} \mathrm{~s}^{-1}$ which is the one immersed in the molecular cloud, and is absent in those immersed in the atomic gas $\left(v>3.8 \mathrm{~km} \mathrm{~s}^{-1}\right)$. This suggests that the $\mathrm{SO}^{+}$emission comes from a more shielded region than that of $\mathrm{CO}^{+}$.

Finally, $\mathrm{C}_{3} \mathrm{H}_{2}$ has been detected towards the star position and the PDR-peak. We have estimated the rotation temperature towards the PDR-peak using the technique of rotational diagrams. Since the size of the emitting region is unknown, we have calculated the rotation temperature in the two limiting cases of a point source and an extended source (beam filling factor $=1$ ). The obtained rotational temperature towards the PDR-peak is of $\sim 8 \mathrm{~K}$ (assuming a point source) and $\sim 15 \mathrm{~K}$ (assuming extended emission) (see Fig. A.1). With these values we estimate a $\mathrm{C}_{3} \mathrm{H}_{2}$ column density of $\sim 1.2-2.1 \times 10^{12} \mathrm{~cm}^{-2}$ and a fractional abundance of $\sim 1-2 \times 10^{-10}$ depending on the assumed source size. We have not detected $\mathrm{C}_{3} \mathrm{H}_{2}$ towards the molecular peak. Assuming an excitation temperature of $15 \mathrm{~K}$, we find an upper limit of $<5 \times 10^{11} \mathrm{~cm}^{-2}$ for the $\mathrm{C}_{3} \mathrm{H}_{2}$ column density and of $<3 \times 10^{-12}$ for the $\mathrm{C}_{3} \mathrm{H}_{2}$ abundance in the molecular-peak, i.e., at least a factor of 100 lower than towards the PDR-peak.

Only the lowest rotational transition of $\mathrm{C}_{3} \mathrm{H}_{2}$ has been detected towards the star position. Assuming a rotation temperature of $15 \mathrm{~K}$ (which is consistent with the upper limits obtained for the integrated intensity of the higher excitation lines), we derive a $\mathrm{C}_{3} \mathrm{H}_{2}$ column density of $4.3 \times 10^{11} \mathrm{~cm}^{-2}$ and a fractional abundance of $\sim 2 \times 10^{-10}$.

Summarizing, the reactive ions $\mathrm{CO}^{+}, \mathrm{HOC}^{+}$and $\mathrm{SO}^{+}$have only been detected towards the PDR peak in NGC 7023. The comparison between the estimated fractional abundances in the PDR peak and the upper limits towards the molecular peak, show that the abundances of these molecular ions are enhanced at least by a factor of $\sim 10$ in the PDR. Observations of $\mathrm{C}_{3} \mathrm{H}_{2}$ in these same positions reveals that the abundance of this carbon chain is a factor of $\sim 100$ larger in the PDR than in the molecular cloud. 


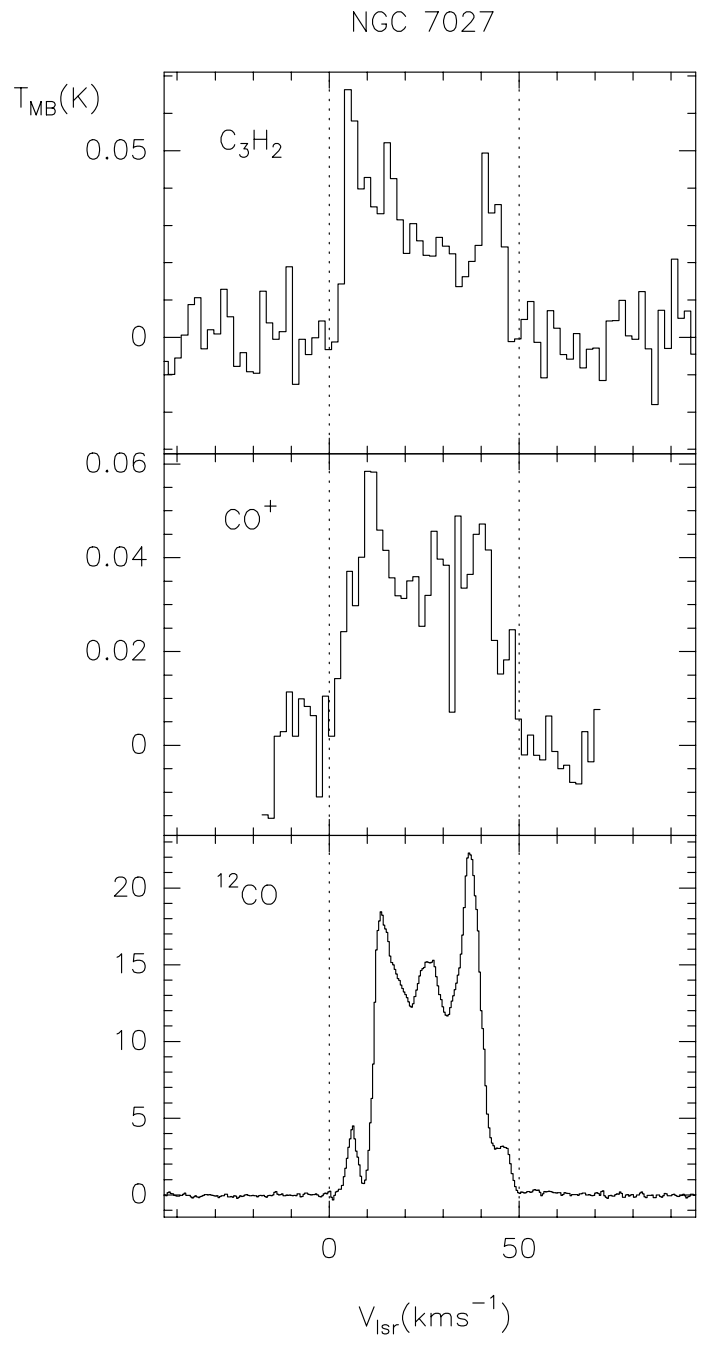

Fig. 6. Spectra of the $\mathrm{C}_{3} \mathrm{H}_{2} \quad 6(1,6) \rightarrow 5(0,5), \mathrm{CO}^{+} 2,5 / 2 \rightarrow 1,3 / 2$ and ${ }^{12} \mathrm{CO} 2 \rightarrow 1$ line towards NGC 7027 . Note the larger linewidth of the $\mathrm{CO}^{+}$and $\mathrm{C}_{3} \mathrm{H}_{2}$ lines compared with that of ${ }^{12} \mathrm{CO}$.

\subsection{The Orion Bar}

Intense $\mathrm{HOC}^{+}$emission has been detected towards the Orion Bar. Rotation temperatures have been derived towards the molecular peak and the offset $(+10,-10)$. The main uncertainty in the derived temperatures comes from the unknown beam filling factor. We have calculated the rotation temperature in the two limiting cases, the point source and extended emission. The derived rotation temperatures range from 6 to $30 \mathrm{~K}$, and seem to increase towards the IF. Close to the IF [offset $(+10,-10)]$, the derived rotation temperature is similar to that found towards the PDR-peak in NGC 7023. $\mathrm{HOC}^{+}$column densities range from 2 to $4 \times 10^{11} \mathrm{~cm}^{-2}$ in the detected positions. Based on the results of Hogerheijde et al. (1995), we assume a total $\mathrm{H}_{2}$ column density of $6.5 \times 10^{22} \mathrm{~cm}^{-2}$ towards the molecular peak and of $6.5 \times 10^{21} \mathrm{~cm}^{-2}$ towards the IF. With these values, the $\mathrm{HOC}^{+}$fractional abundance is $\sim 3-5 \times 10^{-12}$ towards the molecular peak (depending on the assumed source size) and $\sim 3 \times 10^{-11}$ towards the IF. Thus, the fractional abundance of $\mathrm{HOC}^{+}$increases towards the IF by a factor of $\sim 10$.
This increase of the $\mathrm{HOC}^{+}$abundance is accompanied by a decrease of the $\left[\mathrm{HCO}^{+}\right] /\left[\mathrm{HOC}^{+}\right]$ratio. As explained by Woods et al. (1983), in the limit of subthermal excitation the $N \propto T_{\mathrm{R}}$ and the $\left[\mathrm{HCO}^{+}\right] /\left[\mathrm{HOC}^{+}\right]$ratio can be calculated as the ratio of the intensities of the $\mathrm{HC}^{18} \mathrm{O}^{+} 1 \rightarrow 0$ and $\mathrm{HOC}^{+}$ $1 \rightarrow 0$ lines corrected by the ${ }^{16} \mathrm{O} /{ }^{18} \mathrm{O}$ isotopic ratio. We have assumed a value of 650 for the ${ }^{16} \mathrm{O} /{ }^{18} \mathrm{O}$ ratio and obtained the values shown in Table 2 . The $\left[\mathrm{HCO}^{+}\right] /\left[\mathrm{HOC}^{+}\right]$ratio increases from $\sim 400$ in the molecular peak to $<166$ towards the IF. Apponi et al. (1999) estimated a $\left[\mathrm{HCO}^{+}\right] /\left[\mathrm{HOC}^{+}\right] \sim$ 270 towards the Orion Bar and interpreted it as a consequence of the unique chemistry of PDR regions. The higher angular resolution of our observations allow us to detect a gradient in the $\left[\mathrm{HCO}^{+}\right] /\left[\mathrm{HOC}^{+}\right]$ratio with the lowest values towards the IF. This gradient support the interpretation of an enhanced $\mathrm{HOC}^{+}$abundance in the PDR.

The reactive ion $\mathrm{CO}^{+}$has been detected towards the three positions of the strip. The $\mathrm{CO}^{+}$column density peaks towards the offset $\left(+10^{\prime \prime},-10^{\prime \prime}\right)$ instead of towards the maximum in $\mathrm{H}_{2}$ column density [offset $\left(+20^{\prime \prime},-20^{\prime \prime}\right)$ ]. Since the excitation conditions are quite uniform across the Orion Bar, this implies that the $\mathrm{CO}^{+}$abundance is enhanced close to the IF. Taking the same values for the total $\mathrm{H}_{2}$ column density as before, we estimate a $\mathrm{CO}^{+}$fractional abundance of $\sim 1.5 \times 10^{-11}$ towards the molecular peak and $\sim 6.1 \times 10^{-11}$ towards the IF.

The molecular ion $\mathrm{SO}^{+}$has also been detected towards the three observed positions of the Orion-Bar. The $\mathrm{SO}^{+}$column density peaks towards the molecular peak and the derived fractional abundance is $\sim 1.3 \times 10^{-10}$. We estimated a $\mathrm{SO}^{+}$abundance towards the IF of $\sim 1.8 \times 10^{-10}$. Thus, the $\mathrm{SO}^{+}$abundance seems to be quite uniform across the Orion Bar. Simultaneously with $\mathrm{SO}^{+}$, we have observed $\mathrm{SO}_{2}$. Like most molecules (see the $\mathrm{HC}^{18} \mathrm{O}$ data in this paper and the molecular observations reported by Fuente et al. 1996b), the $\mathrm{SO}_{2}$ column density is maximum towards the molecular peak and decrease rapidly towards the IF.

Finally, several lines of the radical $\mathrm{C}_{3} \mathrm{H}_{2}$ have been detected towards all the positions of the strip. Since the emission seems extended we have assumed a beam filling factor of 1 in our calculations of the rotation temperature. The derived rotation temperatures are quite similar in all the observed positions with values $\sim 13-15 \mathrm{~K}$ (see Fig. A.1). This is consistent with previous assumption of uniform excitation conditions across the strip for $\mathrm{CO}^{+}$and $\mathrm{SO}^{+}$. However, the $\mathrm{C}_{3} \mathrm{H}_{2}$ abundance does increase towards the IF. In fact, the fractional abundance of $\mathrm{C}_{3} \mathrm{H}_{2}$ is almost an order of magnitude larger towards the IF, $X_{\mathrm{C}_{3} \mathrm{H}_{2}} \sim 1.4 \times 10^{-9}$, than towards the molecular peak, $X_{\mathrm{C}_{3} \mathrm{H}_{2}}$ $\sim 2.0 \times 10^{-10}$ (see Table 7).

Summarizing, the fractional abundances of $\mathrm{CO}^{+}, \mathrm{HOC}^{+}$ and $\mathrm{C}_{3} \mathrm{H}_{2}$ increases by a factor of $\sim 5-20$ towards the IF (see Table 7). The $\mathrm{SO}^{+}$fractional abundance is quite uniform along the strip suggesting that the $\mathrm{SO}^{+}$emission arises in a more shielded region than that of $\mathrm{CO}^{+}, \mathrm{HOC}^{+}$and $\mathrm{C}_{3} \mathrm{H}_{2}$. These results are in agreement with those obtained towards NGC 7023 and confirm the reactive ions $\mathrm{CO}^{+}$and $\mathrm{HOC}^{+}$and the carbon chain $\mathrm{C}_{3} \mathrm{H}_{2}$ as good tracers of PDRs. 


\subsection{NGC 7027}

As expected, the chemistry of NGC 7027 is different from that of the other two studied PDRs. First of all, the molecular ions $\mathrm{HOC}^{+}$and $\mathrm{SO}^{+}$have not been detected.

We have not detected the $\mathrm{HC}^{18} \mathrm{O}^{+} J=1 \rightarrow 0$ line towards NGC 7027. Thus, we will use previous $\mathrm{HCO}^{+}$observations for our estimates of the $\left[\mathrm{HCO}^{+}\right] /\left[\mathrm{HOC}^{+}\right]$abundance ratio. The high excitation $J=4 \rightarrow 3$ and $J=3 \rightarrow 2 \mathrm{HCO}^{+}$lines have recently been observed by Hasegawa \& Kwok (2001). They derived a $N_{\mathrm{HCO}^{+}} / \Delta v \sim 2.5 \times 10^{12} \mathrm{~cm}^{-2} \mathrm{~km}^{-1} \mathrm{~s}$, a size of $\sim 13^{\prime \prime}$ for the emitting region and expansion velocities $\sim 20 \mathrm{~km} \mathrm{~s}^{-1}$ for both lines. We have derived a 3- $\sigma$ upper limit to the $\mathrm{HOC}^{+}$column density, $N_{\mathrm{HOC}^{+}} / \Delta v<2.4 \times 10^{10} \mathrm{~cm}^{-2} \mathrm{~km}^{-1} \mathrm{~s}$ using our upper limit to the $\mathrm{HOC}^{+} 1 \rightarrow 0$ line emission and assuming the source size and the expansion velocity derived from the $\mathrm{HCO}^{+}$lines (see Table 6). Since the size of the PDR in NGC 7027 is much smaller than the beam at the frequency of the $\mathrm{HOC}^{+} J=1 \rightarrow 0$ line (beam $=29^{\prime \prime}$ ), it is more convenient to use the limit on the $\mathrm{HOC}^{+} J=3 \rightarrow 2$ line to derive the $\left[\mathrm{HCO}^{+}\right] /\left[\mathrm{HOC}^{+}\right]$ratio. Using the $\mathrm{HOC}^{+} 3 \rightarrow 2$ data and with the same assumptions as before we obtain $N_{\mathrm{HOC}^{+}} / \Delta v<5.5 \times$ $10^{9} \mathrm{~cm}^{-2} \mathrm{~km}^{-1} \mathrm{~s}$. This implies that the $\left[\mathrm{HCO}^{+}\right] /\left[\mathrm{HOC}^{+}\right]$abundance ratio is $>450$. This ratio is a factor of 4 larger than that measured in the other PDRs. The beam of the telescope at $268 \mathrm{GHz}$ (beam $=9.5^{\prime \prime}$ ) is smaller than the emitting region, then the $\left[\mathrm{HCO}^{+}\right] /\left[\mathrm{HOC}^{+}\right]$ratio could be somewhat overestimated if the $\mathrm{HOC}^{+}$emission arise mainly in the outer part of the PDR. But this would be a very odd $\mathrm{HOC}^{+}$spatial distribution with $\left[\mathrm{HCO}^{+} / \mathrm{HOC}^{+}\right]$ratio decreasing towards the more shielded regions of the nebula instead of towards the PDR, in contradiction with our results in NGC 7023 and the Orion Bar. As discussed in Sect. 6, we consider that the lack of $\mathrm{HOC}^{+}$is more likely due to the peculiar chemistry of this C-rich PN. The non-detection of $\mathrm{SO}^{+}$and $\mathrm{SO}_{2}$ towards this source also argues in favor of this interpretation.

In Fig. 4 we show the $\mathrm{CO}^{+} 2,5 / 2 \rightarrow 1,3 / 2$ and the $\mathrm{CO} 2 \rightarrow 1$ spectra. Both lines have been observed with similar beams. The $\mathrm{CO}^{+}$emission seems to arise in the high velocity layer of the envelope which is adjacent to the ionized gas, while the bulk of the $\mathrm{CO}$ emission arise in the lower velocity and more extended envelope. In order to determine the abundance of $\mathrm{CO}^{+}$, we have fitted the profile of the ${ }^{12} \mathrm{CO}$ line with a two shellmodel. The derived $\mathrm{CO}$ column densities are $N_{\mathrm{CO}}=5.2 \times$ $10^{16} \mathrm{~cm}^{-2}$ in the high velocity shell and $N_{\mathrm{CO}}=2.1 \times 10^{17} \mathrm{~cm}^{-2}$ in the low-velocity one. Thus, assuming that all the $\mathrm{CO}^{+}$emission arise in the high velocity shell, we estimate a $\left[\mathrm{CO}^{+}\right] /[\mathrm{CO}]$ $\sim 6 \times 10^{-5}$. Using a standard value for the fractional $\mathrm{CO}$ abundance, $X_{\mathrm{CO}}=8 \times 10^{-5}$, we derive a $\mathrm{CO}^{+}$fractional abundance of $\sim 5 \times 10^{-9}$. This value is a factor of 4 larger than that found by Hasegawa \& Kwok (2001) with an LVG excitation model. We do not consider this difference significative taking into account the large uncertainties in these calculations. The collisional excitation of $\mathrm{CO}^{+}$is poorly understood and should be treated simultaneously with its chemistry (Black 1998). On the other hand, the separation between the high-velocity and lowvelocity shells in the $\mathrm{CO}$ line involves some uncertainty.
An intense $\mathrm{C}_{3} \mathrm{H}_{2}$ line has been detected towards this PN. The linewidth of this line is similar to that of $\mathrm{CO}^{+}$and much larger than that of the $\mathrm{CO}$ line. As in the case of the $\mathrm{CO}^{+}$line, we assume that all the emissions arise in the high-velocity shell and derive a $\mathrm{C}_{3} \mathrm{H}_{2}$ fractional abundance, $X_{\mathrm{C}_{3} \mathrm{H}_{2}} \sim 1 \times 10^{-8}$. This high $\mathrm{C}_{3} \mathrm{H}_{2}$ abundance is consistent with the enhanced $\mathrm{C}_{3} \mathrm{H}_{2}$ abundances observed in the other PDRs.

Summarizing, enhanced $\mathrm{CO}^{+}$and $\mathrm{C}_{3} \mathrm{H}_{2}$ fractional abundances are found towards NGC 7027 in agreement with the results found towards NGC 7023 and the Orion Bar. However, $\mathrm{HOC}^{+}$and $\mathrm{SO}^{+}$have not been detected which indicates a different PDR chemistry in the PN.

\section{Discussion: PDR chemistry}

In Table 7, we give a summary of the fractional abundances and abundance ratios observed towards the studied PDRs. The PDRs in the Orion Bar and NGC 7023 share the same chemistry. The fractional abundances of the reactive ions $\mathrm{HOC}^{+}$, $\mathrm{CO}^{+}$and $\mathrm{SO}^{+}$are enhanced towards the PDRs of these regions. The variations of the $\left[\mathrm{HCO}^{+}\right] /\left[\mathrm{HOC}^{+}\right]$ratio across the PDRs prove that these ratios can be used as tracers of the gas exposed to intense UV radiation. Comparing the $\mathrm{CO}^{+}$and $\mathrm{SO}^{+}$results, the latter seems to arise in a more shielded region of the PDR.

The chemistry in NGC 7027 is clearly different. The highest $\mathrm{CO}^{+}$and $\mathrm{C}_{3} \mathrm{H}_{2}$ fractional abundances are measured toward this PN. However, the fractional abundances of the reactive ions $\mathrm{HOC}^{+}$and $\mathrm{SO}^{+}$are at least an order of magnitude lower than in NGC 7023 and the Orion Bar. Although detailed chemical modeling is beyond the scope of this paper, in the following we discuss our results in NGC 7023, the Orion Bar and NGC 7027 in terms of the existing models. Finally we discuss the peculiar chemistry of the PN.

\section{1. $\mathrm{HCO}^{+} / \mathrm{HOC}^{+} / \mathrm{CO}^{+}$}

The molecular ion $\mathrm{CO}^{+}$was the first reactive ion discovered in the interstellar medium (Latter et al. 1993). This ion reacts on virtually every collision with $\mathrm{H}_{2}$ and does not survive in molecular clouds. However, its abundance becomes significant in the hot layers of PDRs (Sternberg \& Dalgarno 1995). Within the PDR, $\mathrm{CO}^{+}$is formed mainly by

$\mathrm{C}^{+}+\mathrm{OH} \rightarrow \mathrm{CO}^{+}+\mathrm{H}$

and destroyed by reactions with $\mathrm{H}_{2}$ to form $\mathrm{HCO}^{+}$(and $\mathrm{HOC}^{+}$), and by dissociative recombination. In regions of high ionization rate, $\mathrm{CO}^{+}$can also be produced by a charge transfer reaction between $\mathrm{CO}$ and $\mathrm{CH}^{+}$, and/or even by direct ionization of CO. Sternberg \& Dalgarno (1995) predicted a $\mathrm{CO}^{+}$abundance $\gtrsim 10^{-10}$ in the $A_{v}<1$ mag region of the PDR. We have detected $\mathrm{CO}^{+}$in all the target PDRs. The estimated fractional abundances in NGC 7023 and the Orion Bar are $\sim 10^{-11}$. These abundances have been calculated assuming that the $\mathrm{CO}^{+}$emission comes from the total $\mathrm{H}_{2}$ column density. If the emission arises mainly in a layer of $A_{v} \sim 1 \mathrm{mag}$, we obtain $\mathrm{CO}^{+}$fractional abundances ranging from $3 \times 10^{-10}$ to $10^{-9}$ in these 
sources. These values are close to the highest $\mathrm{CO}^{+}$abundances predicted by Sternberg \& Dalgarno (1995) and to the $\mathrm{CO}^{+}$abundance measured in NGC $7027, X_{\mathrm{CO}^{+}} \approx 5 \times 10^{-9}$.

A chemically related ion is $\mathrm{HOC}^{+}$. There are two pathways to form efficiently $\mathrm{HOC}^{+}$,

$\mathrm{C}^{+}+\mathrm{H}_{2} \mathrm{O} \rightarrow \mathrm{HOC}^{+}+\mathrm{H}$

$\mathrm{CO}^{+}+\mathrm{H}_{2} \rightarrow \mathrm{HOC}^{+} / \mathrm{HCO}^{+}+\mathrm{H}$.

Its destruction is mainly by reactions with $\mathrm{H}_{2}$ (Smith et al. 2002). The large abundances of $\mathrm{C}^{+}$and $\mathrm{CO}^{+}$in PDRs could result in an enhanced $\mathrm{HOC}^{+}$abundance. Within this scheme, one expects some correlation between low $\left[\mathrm{HCO}^{+}\right] /\left[\mathrm{HOC}^{+}\right]$ratios and high $\mathrm{CO}^{+}$abundances (Savage et al. 2000). Both quantities seem correlated in NGC 7023 and the Orion Bar. In both regions, the $\mathrm{CO}^{+}$abundance is maximum and the $\left[\mathrm{HCO}^{+}\right] /\left[\mathrm{HOC}^{+}\right]$is minimum towards the PDR-peak. Moreover, the spatial distribution of $\mathrm{HOC}^{+}$and $\mathrm{CO}^{+}$is quite similar across the Orion Bar.

NGC 7027 is the exception to this rule. We have measured the maximum value of the $\mathrm{CO}^{+}$abundance towards this $\mathrm{PN}$. However the $\left[\mathrm{HCO}^{+}\right] /\left[\mathrm{HOC}^{+}\right]$ratio is not as low as observed in NGC 7023 and the Orion Bar. This difference can be understood in terms of the peculiar chemistry of this PN and the $\mathrm{HOC}^{+}$and $\mathrm{CO}^{+}$formation paths. Recent observations with the Infrared Space Observatory (ISO) reveal that the light radicals $\mathrm{OH}, \mathrm{CH}^{+}$and $\mathrm{CH}$ are quite abundant in this PN (Liu et al. 1996, 1997; Cernicharo et al. 1997). However, a very low abundance of $\mathrm{H}_{2} \mathrm{O}, \mathrm{X}_{\mathrm{H}_{2} \mathrm{O}}<10^{-8}$, is observed. The low $\mathrm{H}_{2} \mathrm{O}$ abundance precludes the production of $\mathrm{HOC}^{+}$via reaction (2). The lack of correlation between the abundances of $\mathrm{HOC}^{+}$and $\mathrm{CO}^{+}$in this source suggests that reaction (2) is the dominant $\mathrm{HOC}^{+}$formation path which is inhibited in this C-rich PN.

\section{2. $\mathrm{SO}^{+}$}

$\mathrm{SO}^{+}$has been detected in warm and cold clouds with abundances ranging from $\sim 10^{-9}-10^{-11}$. These fractional abundances can be explained by gas phase ion-molecule chemistry (Turner 1994). $\mathrm{SO}^{+}$is primarily formed via $\mathrm{S}^{+}+\mathrm{OH} \rightarrow \mathrm{SO}^{+}+\mathrm{H}$ and removed by dissociative recombination in $\mathrm{S}+\mathrm{O}$. The neutral atoms $S$ will then follow a series of reactions to give $\mathrm{SO}$ and $\mathrm{SO}_{2}$. In regions exposed to high $\mathrm{UV}$ fields, the sulfur is mainly in the form of $\mathrm{S}^{+}$and $\mathrm{SO}^{+}$, while in the well shielded cloud it is in the form of $\mathrm{SO}$ and $\mathrm{SO}_{2}$. Thus, gradients in the $\left[\mathrm{SO}^{+}\right] /[\mathrm{SO}]$ and $\left[\mathrm{SO}^{+}\right] /\left[\mathrm{SO}_{2}\right]$ abundance ratios are expected across PDRs.

We have detected $\mathrm{SO}^{+}$towards the PDRs in NGC 7023 and the Orion Bar. The $\mathrm{SO}^{+}$abundance is at least an order of magnitude larger towards the PDR peak than towards the molecular cloud in NGC 7023. The $\mathrm{SO}^{+}$abundance towards the PDR peak is in agreement with that predicted by Sternberg \& Dalgarno (1995) in the layers with $A_{v}<2$ mag of a high density PDR. In this case, an enhanced $\mathrm{SO}^{+}$abundance is the result of the large abundance of $\mathrm{S}^{+}$and $\mathrm{OH}$ in the $\mathrm{PDR}$. The $\mathrm{SO}^{+}$abundance towards the Orion Bar remains quite uniform along the observed strip. However, we detect a gradient in the $\left[\mathrm{SO}^{+}\right] /\left[\mathrm{SO}_{2}\right]$ abundance ratio (see Table 7) due very likely to the rapid destruction of $\mathrm{SO}_{2}$ by the $\mathrm{UV}$ radiation.
In both sources, NGC 7023 and the Orion $\mathrm{Bar}, \mathrm{SO}^{+}$seems to arise in a more shielded region of the cloud than $\mathrm{CO}^{+}$. $\mathrm{SO}^{+}$is not as rapidly destroyed by reactions with $\mathrm{H}_{2}$ as $\mathrm{CO}^{+}$. For this reason, $\mathrm{SO}^{+}$is detected in dark clouds while the emission of $\mathrm{CO}^{+}$is restricted to the $\mathrm{HI} / \mathrm{H}_{2}$ transition layer of PDRs. Within the $\mathrm{PDR}, \mathrm{SO}^{+}$is expected to survive in regions with large extinction.

$\mathrm{SO}^{+}$has not been detected in NGC 7027 with an upper limit to the $\left[\mathrm{SO}^{+}\right] /\left[\mathrm{CO}^{+}\right]$abundance ratio of $<0.1$. Values of the $\left[\mathrm{SO}^{+}\right] /\left[\mathrm{CO}^{+}\right] \gg 1$ are observed in NGC 7023 and the Orion Bar. As discussed in Sect. 6.4, this difference can be explained by the different elemental abundances and high electron densities in the PN.

\section{3. $\mathrm{C}_{3} \mathrm{H}_{2}$}

Intense $\mathrm{C}_{3} \mathrm{H}_{2}$ lines have been detected towards all the target sources. The spatial distribution of the emission in NGC 7023 and the Orion Bar shows enhanced $\mathrm{C}_{3} \mathrm{H}_{2}$ abundances towards the PDR. In fact, the $\mathrm{C}_{3} \mathrm{H}_{2}$ abundance is larger by almost a factor of 100 towards the PDR peak than towards the molecular peak in NGC 7023. In the Orion Bar, the $\mathrm{C}_{3} \mathrm{H}_{2}$ abundance is a factor of 10 larger towards the IF than towards the maximum in molecular column density. Thus, we can conclude that most of the $\mathrm{C}_{3} \mathrm{H}_{2}$ emission arises in the PDR of these sources.

We have estimated $\mathrm{C}_{3} \mathrm{H}_{2}$ abundances ranging from $\sim 10^{-10}-10^{-8}$ in our sample. These abundances are similar to those found in dense dark clouds (Cox et al. 1989). Taking into account the large photodissociation rate of $\mathrm{C}_{3} \mathrm{H}_{2}$ (van Dishoeck 1988), a rapid formation is necessary to explain the large $\mathrm{C}_{3} \mathrm{H}_{2}$ abundances found in PDRs. Enhanced abundances of the $\mathrm{CH}_{n}, \mathrm{CH}_{n}{ }^{+}$are produced in PDRs via reactions of the carbon ion $\mathrm{C}^{+}$and $\mathrm{H}_{2}$ (Sternberg \& Dalgarno 1995; Hasegawa et al. 2000). The formation of more complex hydrocarbons like $\mathrm{C}_{3} \mathrm{H}_{2}$ are ruled by reactions of the atomic carbon ion $\mathrm{C}^{+}$with acetylenes to form carbon cations (Bettens et al. 1997). Thus, it requires an acetylene supply. Allain et al. (1996) proposed that acetylene can be the result of the destruction of small PAHs by intense UV fileds. Fossé et al. (2000) compared the Aromatic Infrared Bands (AIBs) emission with the high hydrocarbon abundances towards IC 63 and NGC 7023 and found enhanced carbon chains abundances coincident with the AIBs emission. A similar work has been carried out by Teyssier et al. (2002) in the Horsehead Nebula. They proposed that the high hydrocarbon abundances in PDRs might be explained by the high abundance of the atomic carbon ion $\mathrm{C}^{+}$and the release of acetylene from polycyclic aromatic hydrocarbons (PAHs).

\subsection{NGC 7027: The chemistry of a PN}

The chemistry in a PN is different from that of a PDR in a interstellar cloud. First of all, element abundances are different to the solar values. Secondly, the temperature of the illuminating star $\left(\sim 2 \times 10^{5} \mathrm{~K}\right.$ in the case of NGC 7027) is much higher than the typical temperatures of young OB stars. Finally, time-dependent effects might be important in these rapid evolving objects (Tielens 1993).

NGC 7027 is a C-rich object with a C/O ratio $\sim 1.46$ (Salas et al. 2001). Oxygenated molecules are not expected to be 
abundant in this object. In particular, the low abundance of $\mathrm{H}_{2} \mathrm{O}$ could explain the lack of $\mathrm{HOC}^{+}$in this PN. The element abundance of $S$ is a factor of 2 lower than in the interstellar medium (Salas et al. 2001). The low element abundances of $\mathrm{S}$ and $\mathrm{O}$ could explain the low $\mathrm{SO}^{+}$abundance, with an upper limit to the $\left[\mathrm{SO}^{+}\right] /\left[\mathrm{CO}^{+}\right]$abundance ratio of $0.1 . \mathrm{CO}^{+}$is destroyed rapidly by reactions with $\mathrm{H}_{2}$, but $\mathrm{SO}^{+}$is destroyed mainly by recombination. Therefore, the high electron densities could also favor the low $\left[\mathrm{SO}^{+}\right] /\left[\mathrm{CO}^{+}\right]$abundance ratio. Our results are in agreement with the chemical model of NGC 7027 carried out by Hasegawa et al. (2000) which predicts a $\left[\mathrm{SO}^{+}\right] /\left[\mathrm{CO}^{+}\right] \sim 0.04$. This is a steady state model which uses the standard PDR gas-phase chemistry with the physical conditions and elemental abundances derived for NGC 7027. Time-dependent effects are not required to explain our observations although they could be surely important for a finer-tune of the chemistry.

\section{Summary and conclusions}

We have carried out a survey of reactive ions $\left(\mathrm{CO}^{+}\right.$, $\mathrm{HOC}^{+}, \mathrm{HC}^{18} \mathrm{O}^{+}, \mathrm{SO}^{+}$) towards the three prototypical PDRs, NGC 7023, the Orion Bar and NGC 7027. In addition, we have observed cyclopropenylidene $\left(\mathrm{C}_{3} \mathrm{H}_{2}\right)$ towards the selected PDRs. Our results can be summarized as following:

1. The reactive ion $\mathrm{CO}^{+}$has been detected towards all the targets. Its spatial distribution and kinematics are consistent with the $\mathrm{CO}^{+}$emission arising in the innermost part of the PDR, $A_{v}<2$ mag.

2. The reactive ion $\mathrm{HOC}^{+}$also presents enhanced abundances in PDRs. The $\left[\mathrm{HCO}^{+}\right] /\left[\mathrm{HOC}^{+}\right]$abundance ratio decreases towards the PDRs in NGC 7023 and the Orion Bar respectively. We have derived towards NGC 7023, $\left[\mathrm{HCO}^{+}\right] /\left[\mathrm{HOC}^{+}\right] \sim 50-120$, which is the lowest value of the $\left[\mathrm{HCO}^{+}\right] /\left[\mathrm{HOC}^{+}\right]$ratio measured so far.

3. We have not detected $\mathrm{HOC}^{+}$towards NGC 7027. Interestingly, this is the source with the highest $\mathrm{CO}^{+}$abundance. This result suggests that the chemistry in NGC 7027 is different from that in NGC 7023 and the Orion Bar.

4. The abundance of the sulfuratted ion $\mathrm{SO}^{+}$is enhanced in PDRs. Comparing its spatial distribution and kinematics with those of $\mathrm{CO}^{+}$, we conclude that $\mathrm{SO}^{+}$arises in a more shielded layer than $\mathrm{CO}^{+}$.

5. We have not detected $\mathrm{SO}^{+}$and $\mathrm{SO}_{2}$ in NGC 7027 .

6. The abundance of $\mathrm{C}_{3} \mathrm{H}_{2}$ increase by a factor $10-100$ towards the PDRs in NGC 7023 and the Orion Bar. This carbon chain is also detected with a high abundance $\left(\sim 10^{-8}\right)$ in NGC 7027. Our observations show that the production of this carbon chain is highly enhanced in UV exposed regions.

We have compared our observational results with PDR chemical models. Our conclusions can be summarized as following:

1. The abundances of $\mathrm{CO}^{+}, \mathrm{HOC}^{+}$and $\mathrm{SO}^{+}$can be explained by standard reactions of gas-phase chemistry in a PDR.
2. The lack of correlation between $\mathrm{HOC}^{+}$and $\mathrm{CO}^{+}$in NGC 7027 suggests that the reaction of $\mathrm{C}^{+}+\mathrm{H}_{2} \mathrm{O} \rightarrow$ $\mathrm{HOC}^{+}+\mathrm{H}$ is the dominant path to form $\mathrm{HOC}^{+}$in PDRs.

3. Differences between the chemistry in the star-forming regions NGC 7023 and the Orion Bar and NGC 7027 are very likely due to the peculiar elemental abundances in this C-rich object which differ from solar values and the high electron densities of the PN.

4. The high abundances of $\mathrm{C}_{3} \mathrm{H}_{2}$ cannot be explained by gasphase chemistry. Release of acetylene from PAHs is proposed as the cause of the enhanced production of $\mathrm{C}_{3} \mathrm{H}_{2}$ in PDRs.

Acknowledgements. This paper has been partially funded by the Spanish MCyT under projects DGES/AYA2000-927, ESP2001-4519PE and ESP2002-01693, and European FEDER funds.

\section{Appendix A: Column density estimates}

\section{A.1. $\mathrm{HOC}^{+}$}

The $\mathrm{HOC}^{+}$column density have been calculated assuming optically thin emission and a unique rotation temperature for all energy levels using the following expression

$N=1.94 \times 10^{3} v^{2} W Q\left(T_{\mathrm{r}}\right) \exp \left(E_{u} / K T_{\mathrm{r}}\right) /\left(g_{u} A_{i j}\right)$

where $v$ is the line frequency in $\mathrm{GHz}, W$ is the integrated intensity of the $J=1 \rightarrow 0$ line in $\mathrm{K} \mathrm{kms}^{-1}, A_{i j}$ the Einstein spontaneous emission coefficient in $s^{-1}\left(A_{i j}=2.18 \times 10^{-5} \mathrm{~s}^{-1}\right)$, and $Q\left(T_{\mathrm{r}}\right)$ is the partition function of $\mathrm{HOC}^{+}$at the temperature $T_{\mathrm{r}}$.

When two rotational transitions $(J=1 \rightarrow 0$ and $3 \rightarrow 2)$ have been detected, we have estimated the rotation temperature using the technique of rotational diagrams. In Orion and NGC 7023, we obtain typically rotation temperatures of $\sim 8 \mathrm{~K}$ (assuming a point source) to $\sim 30 \mathrm{~K}$ (assuming extended emission) towards all positions (see Table 2). Column densities have been calculated for these two limiting cases. For the positions in which only one transition has been detected, we have estimated the column density assuming a rotation temperature of $15 \mathrm{~K}$, which is expected to be accurate in a factor of 2 . In NGC 7027 we have assumed a rotation temperature of $\sim 20 \mathrm{~K}$ based on the results of Hasegawa \& Kwok (2001).

\section{A.2. $\mathrm{CO}^{+}$}

$\mathrm{CO}^{+}$has a ${ }^{2} \Sigma$ ground state in which each rotational level is split into two fine-structure levels with $J=N \pm 1 / 2$. The $N=1 \rightarrow 0$ rotational line is heavily obscured by the $\mathrm{O}_{2}$ line at $118 \mathrm{GHz}$ and cannot be observed from ground-based telescopes. The most intense transitions of the $N=2 \rightarrow 1$ rotational spectrum are $N=2 \rightarrow 1, J=5 / 2 \rightarrow 3 / 2$ at $236062.553 \mathrm{MHz}$ and $N=2 \rightarrow 1 J=3 / 2 \rightarrow 1 / 2$ at $235789.641 \mathrm{MHz}$. Column densities of $\mathrm{CO}^{+}$have been calculated using the expression (A.1) with the observational parameters of the $\mathrm{CO}^{+} 2,5 / 2 \rightarrow$ $1,3 / 2$ line. We have assumed a rotation temperature of $10 \mathrm{~K}$ based on the results of Fuente \& Martín-Pintado (1997). The partition function of $\mathrm{CO}^{+}$at $10 \mathrm{~K}$ is 7.77 and Einstein spontaneous emission coefficient of this transition $A_{i j}=4.7 \times 10^{-4}$. 

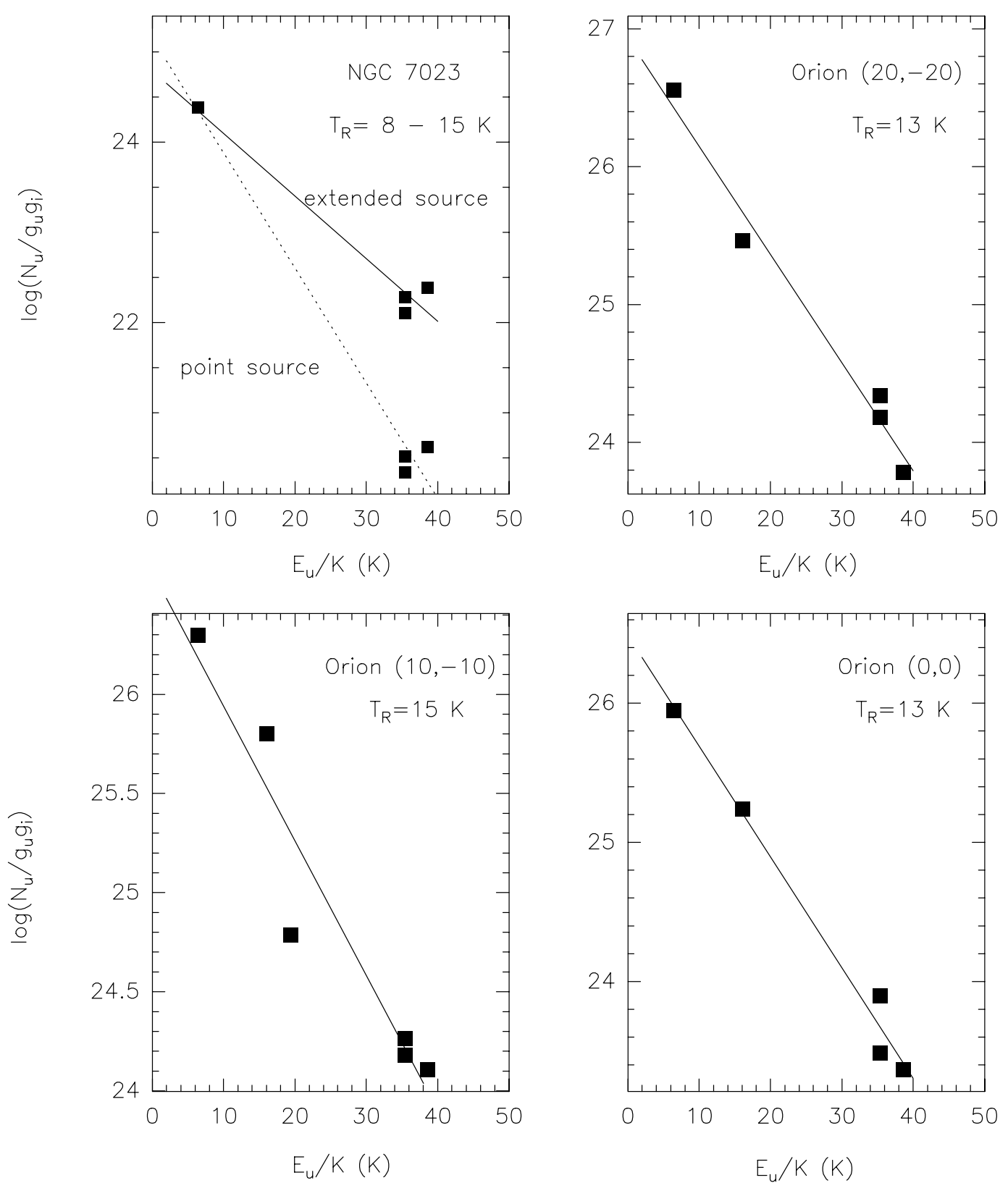

Fig. A.1. Rotational diagrams and fitted values of the rotation temperatures towards the positions where several $\mathrm{C}_{3} \mathrm{H}_{2}$ lines have been observed.

\section{A.3. $\mathrm{SO}^{+}$}

$\mathrm{SO}^{+}$is a reactive radical with a ${ }^{2} \pi_{1 / 2}$ ground state, whose spindoubled rotational transitions are split by $\Gamma$-type doubling. We have observed the $\Gamma$-type doublet of the $J=9 / 2 \rightarrow 7 / 2$ rotational transition at the frequencies of $208590.021 \mathrm{MHz}$ and 208965.425 MHz. We have used the observational parameters of the $208590.021 \mathrm{MHz}$ for our estimates. Column densities have been calculated using the expression (A.1). The Einstein spontaneous emission coefficient of the transition at $208590.021 \mathrm{MHz}$ is $A_{i j}=2.48 \times 10^{-4}$. Since $\mathrm{SO}^{+}$and $\mathrm{CO}^{+}$ have similar dipole moments $\left(\mu=2.77 \mathrm{D}\right.$ for $\mathrm{CO}^{+}, \mu=2.3 \mathrm{D}$ for $\mathrm{SO}^{+}$), we assume the same rotation temperature for $\mathrm{SO}^{+}$as for $\mathrm{CO}^{+}, T_{\mathrm{r}}=10 \mathrm{~K}$. The partition function has been calculated using the expression of the energy levels of a ${ }^{2} \pi_{1 / 2}$ molecule in a Hund's coupling case (a). The ${ }^{2} \pi_{3 / 2}$ ladder has been ignored in the calculation of the partition function since its lowest level lies $525 \mathrm{~K}$ above ground. The partition function at $10 \mathrm{~K}$ is 18.7 .

\section{A.4. $\mathrm{SO}_{2}$}

$\mathrm{SO}_{2}$ is an asymmetric top rotor with constants $A=$ $60778.5511 \mathrm{MHz}, B=10317.96567 \mathrm{MHz}$ and $C=$ $8799.80750 \mathrm{MHz}$. Column densities have been derived from the expression (A.1) with $A_{i j}=6.71 \times 10^{-5}$. Since this is a molecule with large dipole moment $(\mu=1.63 \mathrm{D})$, we have assumed a rotation temperature of $10 \mathrm{~K}$. The partition function has been calculated using the expression

$Q\left(T_{\mathrm{r}}\right)=\left(\frac{5.34 \times 10^{6}}{2} \frac{T_{\mathrm{r}}^{3}}{A B C}\right)^{0.5}$ 


\section{A.5. $\mathrm{C}_{3} \mathrm{H}_{2}$}

The spectrum of cyclopropenylidene is that of an oblate asymmetric top $(\kappa=+0.69)$ with b-type transitions and orthopara symmetry species with relative statistical weights of $3: 1$. Towards the positions where the emission was intense, several rotational lines were observed in order to be able to estimate the rotation temperature.

In Fig. A.1, we present the rotational diagrams of $\mathrm{C}_{3} \mathrm{H}_{2}$ in NGC 7023 and the Orion Bar, with $\log \left(N_{u} /\left(g_{u} g_{I}\right)\right)$ given by

$\log \left(\frac{N_{u}}{g_{u} g_{I}}\right)=\log \left(\frac{3 k W}{8 \pi^{3} v S g_{I} \mu^{2}}\right)$

where $g_{I}$ is $3 / 4$ and $1 / 4$ for the ortho- and para- levels respectively, $W$ is the integrated intensity of the line, $v$ is the frequency, $S$ is the line strength and $\mu$ the dipole moment. Fitting a straight line to the rotational diagrams, we obtain rotation temperatures from $\sim 10 \mathrm{~K}$ to $\sim 15 \mathrm{~K}$. Once the rotation temperature is known, we derive the $\mathrm{C}_{3} \mathrm{H}_{2}$ column density from $N_{\mathrm{C}_{3} \mathrm{H}_{2}}=N_{u} /\left(g_{u} g_{i}\right) Q\left(T_{\mathrm{r}}\right) \exp \left(E_{u} / k T\right)$, where $Q\left(T_{\mathrm{r}}\right)$ is the partition function with the definition of $g_{I}$ used here. In NGC 7023 and the Orion Bar, we have assumed a rotation temperature of $15 \mathrm{~K}$ in the positions in which only one line has been detected. In NGC 7027 we have assumed a rotation temperature of $\sim 20 \mathrm{~K}$ based on the results of Hasegawa \& Kwok (2001).

\section{References}

Allain, T., Leach, S., \& Sedlmayr, E. 1996, A\&A, 305, 616

Apponi, A. J., \& Ziurys, L. M. 1997, ApJ, 481, 800

Apponi, A. J., Pesch, T. C., \& Ziurys, L. M. 1999, ApJ, 519, L89

Bettens, R. P. A., \& Herbst, E. 1997, ApJ, 478, 585

Burton, M. G., Hollenbach, D. J., \& Tielens, A. G. G. M. 1990, ApJ, 365,620

Cernicharo, J., Liu, X.-W., González-Alfonso, E., et al. 1997, ApJ, 483, L65

Cox, P., Walmsley, C. M., \& Güsten, R. 1989, A\&A, 209, 382

Cox, P., Maillard, J.-P., Huggins, P. J., et al. 1997, A\&A, 321, 907

Bieging, J. H., Wilner, D., \& Thronson, H. A. 1991, ApJ, 379, 271

Black, J. H. 1998, Faraday Discussions, Royal Chemical Society (UK), 109, 257

Bregman, J., Larson, K., Rank, D., \& Temi, P. 1994, ApJ, 423, 326

Chokshi, A., Tielens, A. G. G. M., Werner, M. W., \& Castelaz, M. W. 1988, ApJ, 334, 803

Fossé, D., Cesarsky, D., Gerin, M., Lequeux, J., \& Tiné, S. 2000, ISO beyond the peaks: The 2nd ISO workshop on analytical spectroscopy, ed. A. Salama, M. F. Kessler, K. Leech, \& B. Schulz, ESA-SP 456

Fuente, A., Mart'ín-Pintado, J., Cernicharo, J., Brouillet, N., \& Duvert, G. 1992, A\&A, 260, 341

Fuente, A., Martín-Pintado, J., Cernicharo, J., \& Bachiller, R. 1993, A\&A, 276, 473

Fuente, A., Martín-Pintado, J., Neri, R., Rogers, C., \& MoriartySchieven, G. 1996, A\&A, 310, 286
Fuente, A., Rodríguez-Franco, A., \& Martín-Pintado, J. 1996b, A\&A, 312, 599

Fuente, A. \& Martín-Pintado, J. 1997, ApJ, 477, 107L

Fuente, A., Martín-Pintado, J., Rodríguez-Franco, A., \& MoriartySchieven, G. D. 1998, A\&A, 339, 575

Gerin, M., Phillips, T. G., Keene, J., Betz, A. L., \& Boreiko, R. T. 1998, ApJ, 500, 329

Giard, M., Bernard, J. P., Lacombe, F., Normand, P., \& Rouan, D. 1994, A\&A, 291, 239

Hayashi, M., Hasegawa, T., Gatley, I., Garden, R., \& Kaifu, N. 1985, MNRAS, 215, 31P

Hasegawa, T., Volk, K., \& Kwok, S. 2000, ApJ, 532, 994

Hasegawa, T. I., \& Kwok, S. 2001, ApJ, 562, 824

Hogerheijde, M. R., Jansen, D. J., \& van Dishoeck, E. F. 1995, A\&A, 204, 792

Jansen, D. J., Spaans, M., Hogerheijde, M. R., \& van Dishoeck, E. F. 1995, A\&A, 303, 541

Latter, W. B., Walker, C. K., \& Maloney, P. R. 1993, ApJ, 419, L97

Latter, W. B., Dayal, A., Bieging, J. H., et al. 2000, ApJ, 539, 783

Liu, X.-W., Barlow, M. J., Nguyen-Q-Rieu, et al. 1996, A\&A, 315, $\mathrm{L} 257$

Liu, X.-W, Barlow, M. J., Dalgarno, A., et al. 1997, MNRAS, 290, L71

Omodaka, T., Hayashi, M., Hasegawa, T., \& Hayashi, S. S. 1994, ApJ, 430, 256

Parmar, P. S., Lacy, J. H., \& Achtermann, J. M. 1991, ApJ, 372, L25.

Rodríguez-Franco, A., Martín-Pintado, J., Gómez-González, J., \& Planesas, P. 1992, A\&A, 264, 592.

Rodríguez-Franco, A., Martín-Pintado, J., \& Fuente, A. 1998, A\&A, 329, 1097.

Rogers, C., Heyer, M. H., \& Dewdney, P. E. 1995, ApJ, 442, 694

Salas, B. J., Pottasch, S. R., Beintema, D. A., \& Wesselius, P. R. 2001, A\&A, 367, 949

Smith, M. A., Schlemmer, S., von Richthofen, J., \& Gerlich, D. 2002, ApJ, 578, L87

Savage, C., Apponi, A. J., Highberger, J. L., \& Ziurys, L. M. 2000, Am. Astron. Soc. Meet., 196, 26.14

Sternberg, A., \& Dalgarno, A. 1995, ApJS, 99, 565

Stöerzer, H., Stutzki, J., \& Sternberg, A. 1995, A\&A, 296, L9

Tauber, J. A., Tielens, A. G. G. A., Meixner, M., \& Goldsmith, P. F. 1994, ApJ, 422, 136

Tauber, J. A., Lis, D. C., Keene, J., Schilke, P., \& Buttgenbach, T. H. 1995, A\&A, 297, 567

Teyssier, D., Fossé, D., Gerin, M., et al. 2002, Proceedings of the conference Chemistry as a Diagnostic of Star Formation, University of Waterloo, Canada, 21-23 August 2002, ed. C. L. Curry, \& M. Fich

Tielens, A. G. G. M. 1993 in Planetary Nebulae, ed. R. Weinberger, \& A. Acker, IAU Symp., 155, 155 (Kluwer)

Turner, B. E. 1992, ApJ, 396, L107

Turner, B. E. 1994, ApJ, 430, 727

van Dishoeck, E. F. 1988, in Rate Coefficients in Astrochemistry, ed. T. J. Millar, \& D. A. Williams

Volk, K., \& Kwok, S. 1997, ApJ, 477, 722

White, G. J., \& Sandell, G. 1995, A\&A, 299, 179

Woods, R. C., Gudeman, C. S., Dickman, R. L., et al. 1983, ApJ, 270, 583

Yusef-Zadeh, F. 1990, ApJ, 361, L19

Ziurys, L. M., \& Apponi, A. J. 1995, ApJ, 455, 73 